\title{
Abstracts from the 2018 Annual Scientific Meeting of the British and Irish Hypertension Society (BIHS)
}

Robinson College, Cambridge

C Springer Nature Limited 2018

24-26 September 2018

Conference Organizer: In Conference Ltd. http:// bihsevents.org/

Presenting author names are underlined in the contributor lists.

Sponsorship: Funding for the publication of this supplement was provided by the British and Irish Hypertension Society for the advancement of knowledge and dissemination of information concerning the pathophysiology, epidemiology, detection, investigation and treatment of arterial hypertension and related vascular diseases.

\section{$0-1$}

$\beta$-catenin promotes cell survival in endothelial cells under disturbed flow through activation of the endothelial nitric oxide synthase (eNOS) and transcription of pro-survival genes like Survivin, Bcl2 and BIRC3

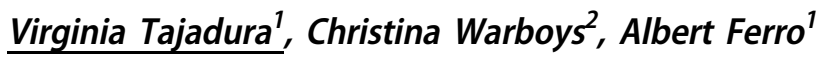 \\ ${ }^{1}$ King's College London, United Kingdom, ${ }^{2}$ Imperial College London, United Kingdom}

Introduction: Endothelial cell apoptosis is associated with the development of atherosclerotic plaques that develop predominantly at sites exposed to low magnitude, multidirectional (disturbed) flow. Therefore, strategies to promote cell survival or prevent apoptosis may be important in reducing cardiovascular disease. In this study, we investigated the role of $\beta$-catenin as a mediator of cell survival in endothelial cells exposed to physiological or disturbed flow.

Methods: Human umbilical vein endothelial cells (HUVEC) were plated in 6-well plates and subjected to flow for $72 \mathrm{~h}$ on an orbital shaker. Drugs to inhibit $\beta$ catenin transcriptional activity were added to cells for the last $24 \mathrm{~h}$ while siRNA was added before exposing EC to flow. Cells where fixed and stained to monitor apoptosis or samples collected from the centre of the well (disturbed flow) or from the edge of the well (undisturbed flow) for mRNA and western blot analysis.

Results: We observed eNOS and $\beta$-catenin interaction in HUVECs exposed to undisturbed flow (UF) and disturbed flow (DF) by proximity ligation assay. $\beta$-catenin siRNA-knockdown cells exposed to flow showed decreased eNOS phosphorylation (Ser1177) but increased apoptosis was only observed in DF exposed EC, suggesting that other $\beta$-catenin-independent mechanisms contribute to cell survival in endothelial cells under UF. The expression of $\mathrm{Bcl}-2$ and survivin, transcriptional targets of B-catenin, was downregulated in DF exposed EC compared to UF and when the cells were treated with specific B-catenin/TCF-LEF inhibitors further reduction of $\mathrm{Bcl}-2$, survivin and BIRC3 expression was observed and apoptosis increased in EC exposed to DF.

Conclusions: $\beta$-catenin is a positive regulator of eNOS activity in HUVEC and $\beta$-catenin-dependent transcription is essential to maintain cell survival in endothelial cells under DF.

Keywords: Nitric Oxide, Beta catenin, Disturbed flow, Apoptosis, Atherosclerosis

Disclosures: none

\section{0 - 2}

Grapefruit Juice (Furancoumarin)-Beetroot Juice (Dietary Nitrate) Cocktail: Enhanced Blood PressureLowering and Taste: Interactions with the NitrateNitrite-NO Pathway through CYP3A4 Inhibition 


\section{of Nitrite Oxidation, and the Enterosalivary Circulation}

\section{Kevin O'Gallagher ${ }^{1}$, Sarah Borg Cardona ${ }^{1}$, Callum Hill ${ }^{1}$, Ali Al-Saedi ${ }^{1}$, Karen McNeill', Charlotte Mills ${ }^{1}$, Andrew James Webb ${ }^{7}$}

\section{${ }^{1}$ King's College London, United Kingdom}

Introduction: Dietary (inorganic) nitrate (NO3-), e.g. beetroot juice (BRJ), lowers blood pressure (BP) via the nitrate-nitrite(NO2-)-nitric oxide(NO) pathway. However, $\mathrm{NO}$ and nitrite are rapidly inactivated via re-oxidation to nitrate, potentially limiting its activity. Cytochrome P4503A4 (CYP3A4)-inhibition with troleandomycin prevents nitrite re-oxidation to nitrate in rodent liver ${ }^{(1)}$. We hypothesized that (golden) grapefruit juice (GFJ) would enhance BP-lowering with Nitrate-BRJ: maintaining circulating [nitrite] through furancoumarin CYP3A4inhibition.

Methods: Following ethical approval, we performed a randomized, placebo-controlled, 7-hour crossover study in 11 fully-consented healthy volunteers, attending on 3 occasions, receiving: Nitrate-BRJ $70 \mathrm{ml}$-shot $\left(\right.$ Beet-It ${ }^{\circledR}$, Heartbeet Ltd, Esher, UK) and either (i) GFJ (250 ml), or (ii) water Buxton $^{\circledast}, 250 \mathrm{ml}$, Buxton Water Ltd, Skelmersdale, UK); or (iii) Placebo-BRJ + GFJ.

Results: Systolic BP (SBP) was decreased by Nitrate$\mathrm{BRJ}+\mathrm{GFJ}$ versus Placebo-BRJ + GFJ $\quad(\mathrm{P}=0.0005$; ANOVA); e.g. by $5.5 \mathrm{mmHg}$ (95\%CI $1.8-9.3)$ at $5.5 \mathrm{~h}$, and relative to baseline by Nitrate-BRJ + GFJ and Nitrate$\mathrm{BRJ}+\mathrm{H} 2 \mathrm{O}$ (both $\mathrm{P}<0.05$ ), but not Placebo-BRJ + GFJ $(\mathrm{P}=0.09)$. Moreover, Nitrate-BRJ + GFJ lowered SBP versus Nitrate- $\mathrm{BRJ}+\mathrm{H} 2 \mathrm{O}(\mathrm{P}=0.02)$, and pulse pressure $(\mathrm{P}=0.0001)$, e.g. by $4.4 \mathrm{mmHg}(95 \% \mathrm{CI} 0.5-8.4)$ at $4 \mathrm{~h}$, but not diastolic BP (DBP) $(\mathrm{P}=0.2)$. Nitrate-BRJ increased plasma [nitrite] $(\mathrm{P}<0.0001)$ versus Placebo$\mathrm{BRJ}+\mathrm{GFJ}$. However, plasma [nitrite] was lower with nitrate-BRJ + GFJ versus nitrate-BRJ + H2O $(\mathrm{p}=0.01)$, as was salivary nitrite production $(\mathrm{P}=0.001)$ and saliva volume $(0.11 \mathrm{ml} / \mathrm{min}(95 \%$-CI $0.02-0.2))$. The taste score of Nitrate-BRJ + GFJ was $1.8 / 10$ points higher than Nitrate-BRJ $+\mathrm{H} 2 \mathrm{O}(\mathrm{P}=0.04)$.

Conclusions: GFJ enhanced BP-lowering of Nitrate$\mathrm{BRJ}$, and taste, representing a novel interaction/intervention: despite decreased plasma [nitrite]/inhibition of enterosalivary circulation of nitrate/nitrite. Potential alternative kinetic mechanisms include CYP3A4inhibition at the tissue-level (c.f. rodent liver), or a synergistic dynamic effect.

Disclosures: A.W. is a shareholder of HeartBeet Ltd which manufactures the active and placebo Beet-It ${ }^{\circledR}$ used in this study. Other Authors: 'none'.

\section{References:}

1.Curtis E, Hsu LL, Noguchi AC, Geary L, Shiva S. Oxygen regulates tissue nitrite metabolism. Antioxid Redox Signal. 2012;17(7):951-61.

\section{0 - 3}

\section{Mediation by blood pressure and other risk factors of relationships between body composition and cardiac structure in adolescents}

\section{Hannah Taylor ${ }^{1}$, Chloe Park ${ }^{1}$, Abigail Fraser ${ }^{2}$, Laura Howe $^{2}$, Debbie Lawlor', George Davey Smith ${ }^{2}$, Nishi Chaturvedi ${ }^{1}$, Alun Hughes ${ }^{1}$}

${ }^{1}$ Cardiovascular Phenotyping Group, Institute of Cardiovascular Science, University College London, United Kingdom, ${ }^{2}$ MRC Integrative Epidemiology Unit, University of Bristol, United Kingdom

Introduction: Fat and lean mass affect cardiac health but the extent to which associations are mediated by other cardiovascular risk factors is unclear. We investigated mediation by blood pressure (BP) and other biomarkers in associations between body composition and left ventricular mass (LVM) or relative wall thickening (RWT) in the Avon Longitudinal Study of Parents and Children.

Methods: Body composition was assessed by dual energy X-ray absorptiometry (DXA) in 2,068 individuals (age 17.7(SD 0.32) years; 45\% male; weight 67(SD 13) $\mathrm{kg})$. BP was measured and echocardiography and blood assays performed. Associations between body composition and RWT and LVM, and the extent of mediation by individual risk factors was estimated by structural equation modelling. Height, sex and socioeconomic position were included as potential confounders in all models.

Results: Path coefficients (standardized $\beta$ ) for associations between fat and lean mass and LVM were 0.28 (95\% confidence interval $0.25,0.31)$ and $0.84(0.77,0.90)$ respectively. Table 1 shows direct (i.e. total-indirect [mediated]) and total effects with each risk factor as a potential mediator; mediation was $<10 \%$ in all cases. There were no convincing associations between fat or lean mass and RWT.

Conclusions: BP only accounts for a small part of the effect of fat and lean mass on LVM. Other biomarkers individually make only very minor or negligible contributions. Most of the association between body composition and LV mass appears to be a direct effect.

Disclosures: None 
Table $1[\mathrm{O}-3]$

\begin{tabular}{lllll}
\hline & Fat mass & \multicolumn{2}{c}{ Lean mass } \\
\cline { 2 - 3 } \cline { 5 - 5 } Mediator & $\begin{array}{l}\text { Direct } \\
\text { effect }\end{array}$ & $\begin{array}{c}\text { Total } \\
\text { effect }\end{array}$ & $\begin{array}{l}\text { Direct } \\
\text { effect }\end{array}$ & $\begin{array}{c}\text { Total } \\
\text { effect }\end{array}$ \\
\hline $\begin{array}{l}\text { Mean arterial } \\
\text { pressure }\end{array}$ & 0.27 & 0.28 & 0.83 & 0.84 \\
Pulse pressure & 0.28 & 0.28 & 0.83 & 0.83 \\
Heart rate & 0.30 & 0.28 & 0.80 & 0.82 \\
Insulin & 0.28 & 0.28 & 0.84 & 0.84 \\
HOMA-IR & 0.28 & 0.28 & 0.84 & 0.84 \\
HDL cholesterol & 0.28 & 0.28 & 0.84 & 0.83 \\
Total cholesterol & 0.27 & 0.28 & 0.84 & 0.83 \\
Triglycerides & 0.28 & 0.28 & 0.84 & 0.84 \\
C-reactive protein & 0.28 & 0.28 & 0.84 & 0.83 \\
Glycoprotein & 0.27 & 0.28 & 0.84 & 0.83 \\
acetyls & & & & \\
\hline
\end{tabular}

\section{$0-4$}

The role of pulse pressure in comparison to systolic blood pressure in the prediction of long-term risk of all-cause- and cardiovascular- mortality, and the influence of age on these relationships, amongst hypertensive patients in the ASCOT-legacy study

Thomas Godec ${ }^{1}$, Judith Mackay ${ }^{2}$, Andrew Whitehouse ${ }^{2}$, Andrew Whitehouse ${ }^{1}$, Neil Poulter Neil Poulter $^{3}$, Peter Sever ${ }^{2}$, Ajay Gupta ${ }^{4}$

${ }^{1}$ London School of Hygiene and Tropical Medicine, United Kingdom, ${ }^{2}$ NHLI, Imperial College London, United

Kingdom, ${ }^{3}$ ICTU, Imperial College London, United

Kingdom, ${ }^{4}$ William Harvey Research Institute, Queen Mary

University of London, United Kingdom

Background: Amongst hypertensive patients, it is not well understood how age influences the predictive ability and the relationship of different blood pressure (BP) measures with the risk of cardiovascular (CV) outcomes and death.

Methods: A UK cohort of 8580 hypertensive patients (mean age 64.1 years) who were part of the AngloScandinavian Cardiovascular Outcomes Trial (ASCOT) $[1,2]$, which concluded in 2005, were followed-up for a median duration of 15.7 years since enrolment to the trial. Cox models were developed to explore the relationship between different BP measurements collected at baseline (SBP, DBP and pulse pressure (PP)), when used alone or in combinations, for each outcome: all-cause (AC)- and CV- mortality. All models were adjusted for age, sex, ethnicity, socio-economic status, body-mass index, total-cholesterol, presence of diabetes, previous vascular event, and smoking status. Interactions between each of the 3-BP measures with age were evaluated. Goodness-of-fit and discriminatory statistics were used to compare models.

Results: There were 3282 (38.3\%) deaths, with 1210 of them from CV-related causes. In all models, both SBP and PP had a linear relationship; but DBP showed a Jshaped association. Across all models, the best included DBP and either SBP or PP alone. There were significant interactions with age and SBP, and age and PP: $p<0.001$ and $\mathrm{p}<0.001$, respectively for AC-mortality; and $\mathrm{p}=$ 0.026 and $\mathrm{p}=0.009$, respectively for $\mathrm{CV}$-mortality. When both SBP and PP were together in a model, SBP no longer showed a significant association with AC- or CV- mortality $(\mathrm{p}=0.799$ and $\mathrm{p}=0.483$, respectively). Figure 1 shows that an increase in SBP and PP, when alone in models, is associated with an increased risk of AC- and CV- mortality. However, when compared to each other, PP was found to have an increased strength of association (and hazards per $10 \mathrm{~mm}$ rise) amongst those below 65 years, and SBP was a better predictor amongst those older than 75 years.

Conclusions: Both SBP and PP are significant and independent predictors of AC- and CV-mortality. However, these relationships are both influenced by age. With an increase in age, the effect of increasing SBP and PP is substantially lessened (see Fig. 1). SBP had a slightly weaker association than PP amongst those below 65 years and stronger amongst those older than 75 years.

Clinical Trial Registry: NCT01499511; https://clinica ltrials.gov

Disclosures: None

References

1. Dahlöf B, et al. The Lancet, 2005; Volume 366, No. 9489; p895-906. https://doi.org/10.1016/S0140-6736(05) 67185-1

2. Sever P, et al. The Lancet, 2003; Volume 361, No. 9364; p1149-1158. https://doi.org/10.1016/S0140-6736 (03)12948-0

\section{O - 5 Omitted}

O - 6 Omitted 

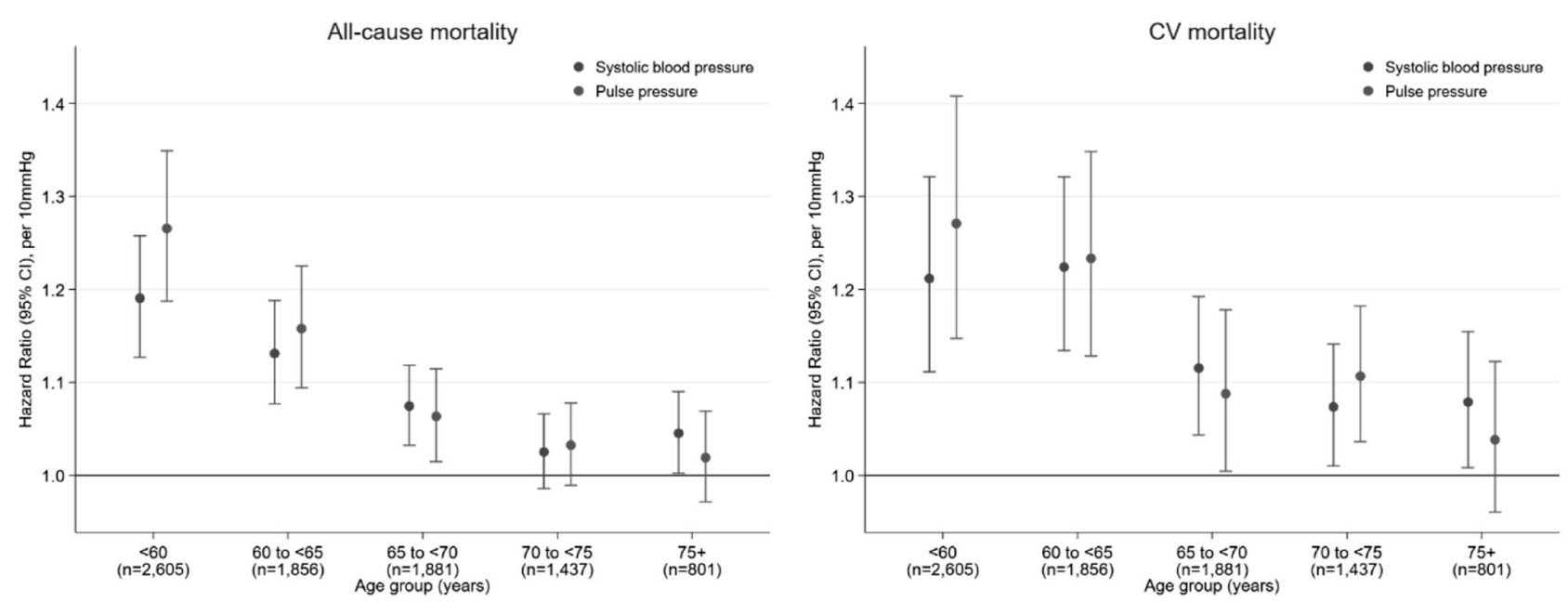

Fig. 1 [O - 4]: Risk (Hazards) of all-cause and cardiovascular- mortality per $10 \mathrm{mmHg}$ rise in systolic BP and pulse pressure, stratified by age (at baseline)

\section{$0-7$}

\section{Maternal risk of fifteen cardiovascular outcomes after pre-eclampsia or gestational hypertension}

\section{Clare Oliver-Williams ${ }^{1}$, David Stevens $^{1}$, Angela Wood ${ }^{1}$ \\ ${ }^{1}$ University of Cambridge, United Kingdom}

Introduction: Hypertensive disorders of pregnancy are a leading cause of maternal morbidity worldwide. Preeclampsia, specifically, is associated with greater cardiovascular disease (CVD) risk. However, the comparative risk of specific cardiovascular outcomes after pre-eclampsia and gestational hypertension is unclear.

Methods: Cox regression analysis compared rates of 15 cardiovascular outcomes in 2,492,313 nulliparous women with a history of pre-eclampsia $(n=95,421)$, gestational hypertension $(n=130,064)$ or normotensive pregnancies $(n$ $=2,287,752$ ), using English electronic health records from women with a first live birth, 1997-2013.

Results: During 20,040,468 person-years of follow-up, the incidence of CVD was 221.56/100 000 person-years [95\%CI, 211.43-232.05], for women with previous preeclampsia, $167.57 / 100000$ person-years [95\%CI, 160.10-175.30], for women with previous gestational hypertension, and was lowest for women with normotensive pregnancies, 82.33/100000 person-years [95\%CI, 81.02-83.65]. Compared with women with normotensive pregnancies, there was greater CVD risk for women with previous gestational hypertension, adjusted $\mathrm{HR}(\mathrm{aHR})=$ $1.58(1.47,1.70), \quad$ or pre-eclampsia, $\quad$ aHR $=2.00$ (1.85-2.16). Previous gestational hypertension was most strongly associated with abdominal aortic aneurysm, aHR $=2.69(1.60-4.54)$, and cardiomyopathy, $\mathrm{aHR}=2.27$
(1.65-3.11). While women with previous pre-eclampsia were at greatest risk of cardiomyopathy, aHR $=3.01$ $(1.80-5.03)$ and acute myocardial infarction, $\mathrm{aHR}=2.94$ $(2.10,4.11)$.

Conclusions: This is the first study to compare the risk of a spectrum of CVD events for women with pre-eclampsia or gestational hypertension. It demonstrates increased cardiovascular risk that varies by outcome and exposure. Considering the ease of evaluating obstetric history, this concept appears promising as an adjunct measure of CVD risk.

Keywords: Gestational hypertension, pre-eclampsia, cardiovascular disease, women, pregnancy

Disclosures: None

\section{0 - 8}

\section{Neonatal and paediatric outcomes following in utero antihypertensive exposure}

\section{Catherine Fitton ${ }^{1}$, Michael Fleming ${ }^{2}$, Markus Steiner ${ }^{1}$, Lorna Aucott ${ }^{1}$, Jill Pell ${ }^{2}$, Daniel Mackay' ${ }^{2}$, James McLay ${ }^{1}$ \\ ${ }^{1}$ University of Aberdeen, United Kingdom, ${ }^{2}$ University of Glasgow, United Kingdom}

Introduction: Hypertension during pregnancy is a leading cause of morbidity and mortality for mother and child, often necessitating treatment with antihypertensive medication. A systematic review of published literature has identified a lack of high quality studies assessing in utero antihypertensive exposure and child outcomes ${ }^{(1)}$. 
Methods: We undertook a retrospective linkage study involving 268,711 women who had a live singleton birth between 2010 and 2014 in Scotland.

Results: Increased odds of preterm birth (aOR 3.22; 95\% CI 2.88-3.60), low birth weight (aOR 2.13; 95\% CI 1.81-2.51), emergency caesarean section (aOR 1.46; 95\% CI $1.33-1.60$ ), special needs indicator at 10 days (aOR $1.59 ; 95 \%$ CI 1.16-2.16) and 6-8 weeks (aOR 1.64; 95\% CI 1.19-2.25) and circulatory defects (aOR 1.91; 95\% CI 1.29-2.83) were associated with in utero exposure to an antihypertensive in the 300 days before birth. Circulatory defects were associated with antihypertensive exposure from the 2 nd or 3 rd trimester onwards, indicating the defect may be causing the increase in maternal blood pressure. Increased odds of speech developmental issues (aOR 1.28; 95\% CI 1.07-1.52) at 2.5 years follow up was associated with in utero exposure, however abnormal development of gross motor or fine motor function, vision, hearing, behaviour, social skills, attention and emotion were not associated with in utero exposure.

Conclusions: Due to increased risks of adverse birth outcomes, support for women on antihypertensive medication is required to provide the best care at birth.

Disclosures: None

References:

1. Fitton CA, et al. Journal of hypertension. 2017;35 (11):2123 https://doi.org/10.1097/HJH.0000000000001456

\section{$0-9$}

Microcirculatory changes in low birthweight infants in the first year of life

\section{Muti Goloba ${ }^{1,2}$, Uzma Khan ${ }^{1,2}$, Rajendra Raghuraman $^{1,2}$, Amelia Brown ${ }^{1}$, Monique Klein ${ }^{1}$, Nansi Botros', Duolao Wang ${ }^{3}$, Donovan Duffy', Isaac Manyonda $^{2}$, Tarek Antonios ${ }^{1,2}$}

\section{${ }^{1}$ University of London, United Kingdom, ${ }^{2}$ St George's University Hospitals NHS Foundation Trust, London, \\ United Kingdom, ${ }^{3}$ Liverpool School of Tropical Medicine, United Kingdom}

Introduction: Reduction in capillary density (CD) or capillary rarefaction (CR), is an established hallmark of essential hypertension (EH). Low birthweight (LBW) infants, known to have an increased risk of developing future $\mathrm{EH}$, were unexpectedly found to have a significantly higher $\mathrm{CD}$ at birth compared to normal birthweight (NBW) infants. We therefore hypothesised that there is a microcirculatory window in the 1st year of life of LBW infants, during which a process of capillary 'hyperpruning' occurs which leads to CR.

Methods: The George's Capillary Rarefaction Offspring Study (G-CROS) is a longitudinal, multi-centre study of which 284 infants were NBW, born at term, and 77 were LBW. Intravital microscopy was used to measure functional (basal) and structural (maximal) dermal $\mathrm{CD}$ at birth, 3 months, 6 months and 12 months.

Results: LBW infants showed significant reductions in basal $(\mathrm{M}=-47.01 \mathrm{cap} / \mathrm{field}, \mathrm{p}<0.0001)$ and maximal $(\mathrm{M}=$ -48.01 cap/field, $\mathrm{p}<0.0001) \mathrm{CD}$ between birth and 3 months. LBW infants demonstrated significantly higher percentage reductions in basal (mean difference $=-7.81 \%, \mathrm{p}=0.0194$ ) and maximal (mean difference $=-8.29 \%, \mathrm{p}=0.0361$ ) CD between birth and 3 months when compared to NBW controls.

Conclusions: There seems to be a microcirculatory window in the first 3 months of life during which LBW infants undergo a process of capillary'hyperpruning'. Further follow-up studies are required to investigate the role of $\mathrm{CR}$ and the microcirculation in the pathogenesis of $\mathrm{EH}$ and the mechanisms orchestrating this CR in early life.

Disclosures: The study was funded by the British Heart Foundation.

\section{Q- 10 \\ High dietary salt is associated with increased aortic stiffness independent of blood pressure}

\section{Kathleen Connolly ${ }^{1}$, Mark Butlin' ${ }^{2}$, Bark Spronk ${ }^{3}$, Dana Georgevsky ${ }^{2}$, Carmel M McEneiry', Alberto P Avolio ${ }^{2}$, Ian B Wilkinson ${ }^{1}$}

${ }^{1}$ University of Cambridge, United Kingdom, ${ }^{2}$ Macquarie
University, Sydney, Australia, ${ }^{3}$ Yale University, New
Haven, United States

Introduction: High salt consumption is associated with increased blood pressure (BP) and vascular stiffening, but the mechanism is poorly understood. It is not known whether vascular stiffness is altered by salt directly or if stiffening is secondary to salt-dependent changes in BP. Recent work in hypertensive rats has suggested that linear polyanionic molecules called glycosaminoglycans (GAGs) may be involved. Here, we aim to determine to what extent arterial stiffness is salt-dependent, pressure-dependent, or GAG-dependent.

Methods: 24 Sprague-Dawley rats were given either (1) normal chow ('Control'), (2) high-salt chow ('HS'), or (3) high-salt chow and the antihypertensive amlodipine ('HSTx'). Tail-cuff systolic BP (tSBP) was measured throughout. After 12 weeks, rats were anaesthetised and in vivo aortic pulse wave velocity (aPWV) measured between 60 and $150 \mathrm{mmHg}$, controlled via intravenous infusions of phenylephrine and sodium nitroprusside. Ex vivo aortic elastic modulus (aEm) and aortic GAG content were determined post-mortem. Data were analysed using 
ANCOVA, repeated measures ANOVA, and Pearson's correlation.

Results: After 12 weeks, tSBP was only elevated in HS rats $(+8 \mathrm{mmHg}, \mathrm{p}<0.01)$, while in vivo aPWV was increased in both HS and HSTx rats compared to Controls $(+0.27-0.85 \mathrm{~m} / \mathrm{s}, \quad \mathrm{p}<0.01$ for each $5 \mathrm{mmHg}$ interval). There was no difference in ex vivo aEm or aortic GAG content between groups, and no correlation between aPWV or aEm and aortic GAGs.

Conclusions: In Sprague-Dawley rats, in vivo aPWV was salt-dependent but pressure-independent, and was not associated with changes in mechanical stiffness or aortic GAG content. This suggests that high dietary salt consumption increases vascular stiffness via a pressureindependent mechanism.

Disclosures: None

\section{$0-11$}

\section{The role of dietary potassium in the regulation of blood pressure: a renal perspective}

\section{Mohammed Ahmed ${ }^{1}$, Meena Murthy ${ }^{1}$, Kevin $O^{\prime}$ Shaughnessy ${ }^{7}$}

\section{${ }^{1}$ University of Cambridge, United Kingdom}

Introduction: A potassium $(\mathrm{K}+)$ rich diet is known to have an antihypertensive effect, and the evidence for the role of the nephron in $\mathrm{K}+$ sensing has been reported previously[1]. More recently, the distal convoluted tubule (DCT) cells of the kidney have been shown to regulate salt transport in response to variations in intracellular chloride ([Cl- $] \mathrm{i})$, which is influenced by serum $\mathrm{K}+$ levels ${ }^{[2]}$. Since WNKs are chloride sensors, and affect the WNK4/SPAK/NCC pathway in DCT cells, we created a DCT-like HEK 293 cell line, and examined the effect of extracellular $\mathrm{K}+$ on the membrane potential $(\mathrm{Vm})$ and $[\mathrm{Cl}-] \mathrm{i}$.

Methods: Cell lines were transiently transfected with DCT cell specific ion transporters and channels along with voltage or Cl- -sensitive fluorescent proteins respectively. The effect of varying external $\mathrm{KCl}$ on the $\mathrm{Vm}$ of the cells, and [Cl-]i were measured using fluorescence quenching assays.

Results: The transfected model DCT-like HEK 293 cells showed changes in fluorescence in response to depolarization with an increase in $[\mathrm{K}] \mathrm{o}$, over a physiological range (3-5 mM) (Fig. 1A). This signal was blocked by tertiapin Q, and blockade of Clc-kb channel abolished $\mathrm{Cl}$ - entry in response to membrane depolarization (Fig. 1B).

Conclusions: DCT-like HEK 293 cells exhibited responses to varying $[\mathrm{K}] \mathrm{o}$ levels, and corresponding changes in [Cl-]i, similar to that seen in the kidney. We showed this in vitro system is a good model to elucidate the molecular mechanisms involved in balancing $\mathrm{Na}+$ and $\mathrm{K}+$ in the kidney, thus providing a better understanding of how dietary $\mathrm{K}+$ affects blood pressure.

Disclosures: None

\section{References:}

1. Young DB (1985) Analysis of long-term potassium regulation. Endocrine reviews 6 (1):24-44. https://doi.org/ 10.1210/edrv-6-1-24

2. Terker AS, Zhang C, Erspamer KJ, Gamba G, Yang CL, Ellison DH (2016) Unique chloride-sensing properties of WNK4 permit the distal nephron to modulate potassium homeostasis. Kidney international 89 (1):127-134. https:// doi.org/10.1038/ki.2015.289

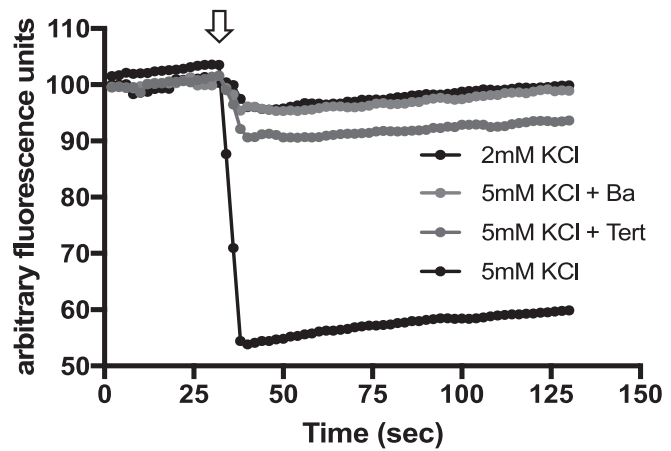

Fig. 1A Change in fluorescence with increasing $\left[\mathrm{K}^{+}\right]_{\mathrm{o}}$ in response to change in $\mathrm{V}_{\mathrm{m}}$

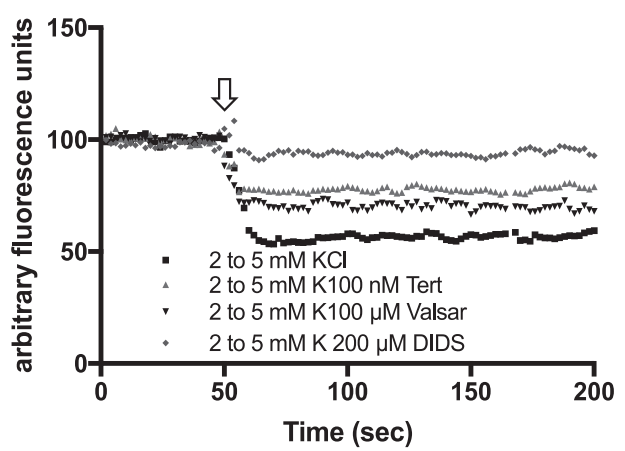

Fig. 1B Effect of various blockers on the fluorescence quenching due to change in $[\mathrm{Cl}] \mathrm{i}$

Fig. 1 [O-11]. (A) Change in fluorescence with increasing $[\mathrm{K}+]_{0}$ in response to change in $\mathrm{Vm}$. (B) Effect of various blockers on the fluorescence quenching due to change in $[\mathrm{Cl}-] \mathrm{i}$ 


\section{$0-12$}

\section{Cholesterol-rich microdomains negatively regulate vascular redox signalling-role of Nox and DJ-1}

\section{Aikaterini Anagnostopoulou ${ }^{1}$, Augusto Montezano ${ }^{1}$, Rhian Touyz}

${ }^{1}$ BHF Cardiovascular Research Center/ University of Glasgow, Glasgow, United Kingdom

Introduction: We hypothesised that Cholesterol-rich microdomains (CRM) are critically involved in regulating cellular redox signalling through processes involving Nox and anti-oxidants, such as PARK7/DJ1 in vascular smooth muscle cells (VSMCs).

Methods: Small arteries and primary VSMCs isolated from human subjects and from WT and Caveolin-1 knockout (Cav1-/-) mice infused with Angiotensin II or vehicle, were studied. Human VSMCs were stimulated with/without Ang II (10-7 M) for 5 or $15 \mathrm{~min}$ in the presence/absence of disrupters (MCD: methyl-b-cyclodextrin; Nys: nystatin) of CRM and processed for sucrose gradient fractionation (lipid-raft isolation); Co-IP; lucigenin (ROS measurement) and immunoblotting.

Results: Human small arteries express Nox5, Nox1, Caveolin-1, total and irreversible oxidised form of DJ1. In fractionated VSMCs, Nox1, Nox5 and total DJ1 but not Nox4 and irreversible oxidised form of DJ1 localised in CRM fractions. Co-IP experiments showed that Caveolin1 interacts with Nox1 and DJ1 but not Nox5; Nox1 interacts with DJ1 in VSMCs. Ang II stimulation and CRM disruptors increased ROS generation (Ang II, 15': $233.8 \pm 83.31 \%$, MCD: $465.2 \pm 151.5 \%$ vs Veh, $\mathrm{p}<0.05$ ); increased phospho-MLC20 and phospho-Ezrin-RadixinMoesin ( $p<0.05$ vs Veh) in human VSMCs. These effects were reversed by cholesterol repletion. Mesenteric arteries from Ang II-infused and Cav1-/- mice exhibited increased ROS generation (Ang II: 330.7 $\pm 54.75 \%$; Cav1-/-: $443.02 \pm 131.70 \%$ vs Control, $\mathrm{p}<0.05)$. Aortas from Ang II-infused and Cav1-/- mice exhibited increased phosphoEzrin-Radixin-Moesin, phospho-ERK1/2 and irreversible oxidised DJ1 ( $\mathrm{p}<0.05$ vs Control).

Conclusions: Cholesterol-rich microdomains act as negative regulators of redox signalling in VSMCs. This could be through inactivation of NOXs or increase of antioxidant activity through DJ1.

Disclosures: None

$$
\begin{aligned}
& \text { O - } 13 \text { Omitted } \\
& \text { O - } 14 \text { Omitted } \\
& \text { O - 15 Omitted }
\end{aligned}
$$

$0-16$

Rapid treatment of Grade 2-3 hypertension in previously untreated patients is accompanied by an increase in total and perfused skin capillary density

Angela C Shore ${ }^{1,2}$, Andrew SP Sharp ${ }^{1,2,3}$, Christine Anning $^{2,3}$, Nicola Pamphilon ${ }^{2,3}$, Claire Ball ${ }^{2,3}$, Christopher Clark ${ }^{1,2}$, Nicholas G Bellenger ${ }^{1,2,3}$, Andrew Jordan $^{1,2,3}$

${ }^{1}$ University of Exeter Medical School, United Kingdom,

${ }^{2}$ NIHR Exeter Clinical Research Facility, United Kingdom,

${ }^{3}$ Royal Devon and Exeter Hospital, United Kingdom

Introduction: Reduced skin capillary density (rarefaction) occurs in patients with, or at risk of, essential hypertension and can be reversed by 6 months antihypertensive medication in mild hypertension [1].

Aim of this study: To assess whether capillary numbers increased in treatment naïve individuals with grade $2-3$ hypertension undergoing a short intensive 18 week treatment regime.

Methods: 54 treatment naive individuals (18-80 years), (office systolic $\mathrm{BP} \geq 170 \mathrm{mmHg}$ and ambulatory daytime average systolic $\mathrm{BP} \geq 150 \mathrm{mmHg}$ ), completed 18 weeks of stepwise treatment intensification every $2-4$ weeks if office BP remained $\geq 140 / 90 \mathrm{mmHg}$. At week 0 and 18 weeks skin capillary density was measured, by intravital capillaroscopy, on the dorsal finger skin at rest and following venous occlusion to measure total capillary numbers.

Results: Treatment increased resting capillary number from 83.8 mean (24.1 SD) to 92.2 (23.6) caps $/ \mathrm{mm} 2 \mathrm{p}=$ 0.004), total capillary number from 104.4(23.8) to 111.5 (23.3) caps $/ \mathrm{mm} 2 \mathrm{p}=0.003$. Capillary reserve was not altered by treatment, \%change of capillary number from resting levels to post occlusion, 27.3 (21.4) \% pre versus $23.6(18.0) \% \mathrm{p}=0.291$ post treatment. Rarefaction was associated with higher plasma glucose and HbA1c $(\mathrm{r}=$ -0.374 and $r=-0.383 ; p<0.006)$, higher LVM $(r=$ $-0.512 \mathrm{p}<0.001)$. Capillary density pretreatment was not associated with the change in systolic or diastolic BP with treatment but correlated with the change in $\mathrm{LVM} \mathrm{r}=0.41$; $\mathrm{p}=0.005$.

Conclusions: Skin capillary density is increased in patients with grade $2-3$ hypertension by a short, 18 week, period of intensive adjustment of blood pressure sufficient to bring $69 \%$ of patients to target office blood pressure.

Disclosures: none

References:

1.Kaiser SE et al; Microcirculation 2013;20(8):703-716 https://doi.org/10.1111/micc.12067 
$0-17$

Altered vascular function in boys with hypospadiasrole of reactive oxygen species

\section{Angela Lucas-Herald ${ }^{1}$, Rheure Alves-Lopes ${ }^{1}$, Augusto Montezano ${ }^{1}$, Syed Faisal Ahmed', Rhian Touyz'; \\ ${ }^{1}$ University of Glasgow, United Kingdom}

Introduction: Hypospadias in boys may be associated with a lack of androgen exposure during the masculinisation programming window. As testosterone has effects on the vasculature, we assessed whether boys with hypospadias have vascular dysfunction.

Methods: Excess foreskin tissue was obtained from boys undergoing hypospadias repair (cases) and circumcision (controls) and small arteries dissected from this tissue. Vascular contractility was assessed by wire myography in response to U46619. VSMCs were cultured and generation of ROS was measured by amplex red and chemiluminescence. Nox mRNA expression was measured by qPCR.

Results: Arteries from cases demonstrated increased constriction to U46619 compared to controls (Emax: 175.6 vs $66.3 \mathrm{p}<0.001$ ), an effect inhibited by $\mathrm{N}$-acetylcysteine. VSMC superoxide anion (5.3 fold) production and $\mathrm{H} 202$ (3.3 fold) levels were increased in cases compared to controls $(\mathrm{p}<0.05)$. Nox5 expression was increased in cases $(2.6$ fold, $\mathrm{p}<0.05$ ). Exposure of vessels to testosterone increased vasoconstriction to U46619 (Emax: 66.3 to $124.6 \mathrm{p}<0.001$ ) in controls, but not in cases. Incubation with NAC abolished the testosterone-induced vascular effects.

Conclusions: In summary, vascular hypercontractility in boys with hypospadias was associated with reduced endothelium-dependent and -independent vasorelaxation, compared with controls. These novel data, from a unique cohort of patients, demonstrate that small arteries from boys with hypospadias exhibit increased vascular contractility and decreased vasorelaxation compared to controls. The functional significance and long-term consequences of vascular dysfunction in androgen-deficient boys is unclear, but may play a role in altered cardiovascular risk later in life.

Disclosures: None
P - 01

Ch-ch-ch-changes-how do patients feel about selftitration of antihypertensive medication?

\section{Lottie Till', Emma Bray', Shelia Greenfield ${ }^{3}$, Sayeed Haque $^{4}$, Richard Hobbs ${ }^{5}$, Paul Little ${ }^{6}$, Jonathan Mant ${ }^{7}$, Bryan Williams ${ }^{8}$, Richard McManus ${ }^{5}$, Claire Schwartz ${ }^{5}$}

${ }^{1}$ University of Oxford, United Kingdom, ${ }^{2}$ School of Nursing, University of Central Lancashire, Preston, United

Kingdom, ${ }^{3}$ Institute of Applied Health Research, University of Birmingham, United Kingdom, ${ }^{4}$ Institute of Clinical

Science, University of Birmingham, United Kingdom,

${ }^{5}$ Department of Primary Care Health Sciences, University of Oxford, United Kingdom, ${ }^{6}$ School of Medicine, University of Southampton, United Kingdom, ${ }^{7}$ Department of Public Health \& Primary Care, University of Cambridge, United Kingdom, ${ }^{8}$ Institute of Cardiovascular Sciences, University College London, United Kingdom

Introduction: Self-management (self-monitoring with selftitration) of hypertension has been shown to lead to better control of blood pressure (BP), but little is known about how patients feel about adjusting their own medications. This study aimed to elucidate the views and concerns of those who self-titrated antihypertensive medication.

Methods: This is a secondary analysis of data from TASMIN-SR ${ }^{(1)}$, a primary care based, randomised trial of self-management of hypertension (involving patients' selfmonitoring their $\mathrm{BP}$, and making medication changes accordingly) compared to usual care. Each time patients changed an antihypertensive medication during the trial open comments were collected from case report forms describing their thoughts and feelings. These were analysed thematically.

Results: The 154/220 (70 \%) self-management patients, who attended the final 12-month follow up, continued to send back information until the end of the study. Five main themes were identified around self-management: motivations to change medications, barriers and exceptions to changing medications, alternatives to medication changes and both positive and negative effects of self-titration. Overall self-titration was seen as empowering, patients liked having a greater say in whether their medication was altered, but they felt that medication increases had to be balanced against side effects and adverse reactions. Many felt more individualised targets were needed, especially those who repeatedly struggled to get their BP to tighter home targets.

Conclusions: The number of antihypertensive medications that a patient can cope with is particularly important given the recent interest for more intensive BP control. Selftitration has the potential to offer a more collaborative way 
for patients to manage antihypertensive medications with their clinician.

Disclosures: None

Reference:

1. isrctn.org Identifier: ISRCTN87171227, https://doi. org/10.1186/ISRCTN87171227

\section{P - 02}

Rapid treatment of moderate-to-severe hypertension using a novel nurse-directed protocol is safe and effective

\section{Andrew Jordan ${ }^{1}$, Christine Anning ${ }^{2}$, Nicola Pamphilon $^{2}$, Lindsay Wilkes ${ }^{2}$, Christopher Clark', Nicholas Bellenger ${ }^{1,3}$, Angela Shore ${ }^{1,2}$, Andrew Sharp $^{1,3}$}

${ }^{1}$ University of Exeter, United Kingdom, ${ }^{2}$ NIHR Exeter Clinical Research Facility, United Kingdom, ${ }^{3}$ Royal Devon and Exeter Hospital NHS Foundation Trust, United Kingdom

Introduction: Protocol-directed therapy may improve the effectiveness of hypertension treatment and control of BP soon after diagnosis of hypertension may improve outcomes. This study assessed for the first time whether 18 weeks' protocol-directed treatment of newly-diagnosed moderate-to-severe hypertension is feasible, safe and effective.

Methods: 55 treatment-naive individuals (18-80 years), (usual care office systolic BP $\geq 170 \mathrm{mmHg}$ and ambulatory daytime average systolic BP $\geq 150 \mathrm{mmHg}$ ), underwent stepwise treatment intensification every $2-4$ weeks if office BP remained $\geq 140 / 90 \mathrm{mmHg}$, with a low threshold to adjust medications if side effects were reported.

Feasibility and acceptability to patients were assessed. Urinary drug metabolites were measured mid-study in treatment-resistant patients and in all participants at 18 weeks. The proportion of patients achieving office $\mathrm{BP}<$ $140 / 90 \mathrm{mmHg}$ at 18 weeks was determined.

Results: A total of 55 participants were recruited, 54 of whom completed the 18 -week protocol. It was feasible to recruit patients from primary care and treatment side effects did not cause any patient to withdraw, with 6 participants having a transient increase in creatinine $>30 \%, 24$ instances of medication intolerance but no episodes of syncope. All side effects resolved upon cessation of causative medication. At 18 weeks, $69 \%$ patients achieved office target BP, (compared to $52 \%$ reported from standard NHS care). Urinary drug metabolites revealed $44 / 48$ (92\%) to be treatment-adherent at 18 weeks.
Conclusions: This study demonstrated, for the first time, that management of treatment-naïve patients with grade 2-3 hypertension using protocol-directed therapy is safe and effective with very high adherence to treatment after 18 weeks.

Disclosures: None

\section{$P$ - 03 \\ The significance of blood pressure in cancellations of elective surgery: an audit of the Hull York medical school region following recent new guidelines}

\section{Rayaan Shaikh ${ }^{1}$, Sarah Carr ${ }^{1}$, Robert O'DonnelI ${ }^{2}$, Terry McCormack $^{1}$ \\ ${ }^{1}$ Hull York Medical School, University of Hull, England, ${ }^{2}$ Royal College of Anaesthetists, Glasgow, Scotland}

Introduction: It is estimated that $>39000$ elective procedures are cancelled annually in the UK due to hypertension $^{1}$. The Measurement of Adult Blood Pressure and the management of hypertension before elective surgery 2016 aimed to address this ${ }^{2}$. This is an AAGBI pilot project designed to assess the impact of these guidelines.

Methods: The three Trusts in the Hull York Medical School Region: York (YTH), Hull and East Yorkshire (HEY) and Northern Lincolnshire and Goole (NLAG) were contacted. Elective surgery data including cancellations were collected for the calendar years 2015 and 2017 and exclusion criteria applied. Cancellation percentages were calculated for each trust and P-values obtained.

Results: YTH $(2.89 \%$ vs. $2.17 \%, \mathrm{p}<0.0001)$ and HEY $(11.02 \%$ vs. $10.52 \%, p=0.016)$ had a statistically significant increase in percentage cancellations in 2017 vs. 2015. At NLAG there was no statistically significant change in cancellations $(4.66 \%$ vs. $4.97 \%, p=0.111)$. There was marked variation in the rate of cancellations between different trusts (3\% YTH, 5\% NLAG and 11\% HEY). It could not be determined if cancellations were due to hypertension, or other factors, as Trusts did not hold this information.

Conclusions: There was a statistically significant increase in elective surgery cancellations at YTH and HEY between 2015 and 2017, although the reasons for this are unclear. Specific reasons for cancellations were not recorded electronically. As part of the upcoming national AAGBI guideline impact assessment, it may therefore be necessary to interrogate patients' notes to identify the reasons for cancellation. Alternatively, it would be useful if the Trusts electronically recorded reasons for cancellations.

Disclosure: None.

References: 
1. Soni S, Chaggar R, Saini R, et al. Unsafe for surgery: a regional survey investigating the variation of pre-operative hypertension management across anaesthetic departments. Anaesthesia 2015; 70 (Suppl 2) p38

2. Hartle A, McCormack T, Carlisle J et al. The measurement of adult blood pressure and management of hypertension before elective surgery 2016. Anaesthesia 2016; 71, p326-337.

\section{$P-04$}

The awareness and implementation of the AAGBI/ BHS Guideline on the Management of Hypertension Before Elective Surgery in the Hull York Medical School Region

\section{Sarah Carr ${ }^{1}$, Rayaan Shaikh ${ }^{1}$, Robert O'Donnell $^{2}$, Terry $\overline{\text { McCormack }}^{1}$}

${ }^{1}$ Hull York Medical School, University of Hull, United

Kingdom, ${ }^{2}$ Royal College of Anaesthetists, Glasgow, Scotland

Introduction: This is an AAGBI guideline impact assessment pilot study for the AAGBI/BHS guideline: The Measurement of Adult Blood Pressure and the Management of Hypertension before Elective Surgery 2016 ${ }^{1}$. This guideline aimed to reduce unnecessary elective surgery cancellations due to hypertension, as there was little evidence to suggest that pre-operative hypertension increased the risk of peri- or post-operative complications ${ }^{2}$.

Methods: We distributed a questionnaire to the consultant anaesthetist responsible for pre-assessment in each trust of the Hull York Medical School region; York (YTH), Hull and East Yorkshire (HEY), and North Lincolnshire and Goole (NLAG).

Results: We received responses from 3 of 3 departments. $100 \%$ of departments surveyed have implemented the guideline, but to different extents; HEY and NLAG circulated the guideline within their anaesthetic departments, whereas YTH has implemented the guideline as departmental policy. Only YTH involved other hospital departments and primary care, but found that GPs do not always provide a recent blood pressure reading. One trust stated that educating medical colleagues has been challenging, possibly limiting the effect of the guideline, but nursing colleagues were more receptive. All respondents stated that the guideline has had some effect on elective surgery cancellations, with the greatest effect being in YTH.

Conclusions: The results of this pilot study indicate a variable response to the introduction of the AAGBI/BHS guideline across NHS trusts. For the upcoming national
AAGBI guideline impact assessment, it would be useful to categorise and code further comments given by respondents in order to allow accurate analysis.

\section{Disclosure: None}

\section{References:}

1. Hartle A, McCormack T, Carlisle J, et al. The measurement of adult blood pressure and management of hypertension before elective surgery. Anaesthesia. 2016; 71 (3): 326-337

2. Soni S, Hartle A. What is a 'safe blood pressure' for anaesthesia? Anaesthesia. 2015; 70(2): 232-233.

\section{P - 05 \\ Limited usefulness of MRI hypertension screening in unselected hypertensives without specific features of a secondary cause}

\section{Catherine Joan Beattie ${ }^{1}$, Punam Pabari ${ }^{1}$, Ben Ariff', Neil Chapman ${ }^{1}$}

\section{${ }^{1}$ Imperial College Healthcare NHS Trust, London, United} Kingdom

Introduction: MR hypertension screening (cardiac, aortic, adrenal and renal MRI) is used in hypertensives as a noninvasive, one-stop investigation to screen for secondary causes and identify left ventricular hypertrophy $(\mathrm{LVH})$. We reviewed local experience of its usefulness as a screening tool in unselected hypertensives without specific features of secondary causes.

Methods: Retrospective review of MRI hypertension screens conducted in a single Trust from January 2013March 2017. Scan reports were reviewed to identify those with LVH and potential secondary causes of hypertension; positive findings were correlated with biochemical results and available clinical information.

Results: 625 MRI screens were completed (377 male [60\%], median age 39 years [range 17-81]). LVH was present in $178(28.5 \%)$. Potential secondary causes of hypertension included aortic coarctation (previously diagnosed, $n=1[0.2 \%])$, renal artery stenosis $(n=15$, including 3 fibromuscular dysplasia [2.4\%]) and other potential renal causes $(n=15 \quad[2.4 \%])$. Adrenal abnormalities were common $(n=173$ [27.7\%] comprising discrete nodules $[n=80 ; 12.8 \%]$ and glands variously reported as 'nodular', 'bulky' or hypertrophied [n $=93 ; 14.9 \%$ ]; among these, biochemical hyperaldosteronism was only confirmed in 11 (6.4\% of those with radiological adrenal abnormalities, $1.8 \%$ of all those screened). No suspected phaeochromocytomas were identified. Incidental findings warranting further investigation were common. 
Conclusions: MRI is a convenient investigation for patients and allows the identification of LVH. However, its use in unselected hypertensive patients (i.e. those without symptoms, signs or biochemical evidence of a specific underlying cause) appears to identify few patients with unsuspected treatable secondary causes and does not obviate the need for appropriate biochemical screening.

Disclosures: None

\section{P - 06}

\section{Hypertension in young adults-A single centre experience}

\section{Matthew Tabinor ${ }^{1}$, Gwyneth Lawson ${ }^{1}$, Madhavan Menon $^{1}$, Richard Fish ${ }^{1}$}

${ }^{1}$ University Hospitals of North Midlands, Stoke-on-Trent, United Kingdom

Introduction: For hypertensive young adults in the UK there is limited data regarding the prevalence of secondary causes. We sought to estimate the proportion of secondary hypertension in adults aged 30 or under referred to our regional specialist hypertension clinic.

Methods: We undertook a retrospective analysis between 01/01/2014 and 31/12/2017. Patients aged 16 to 30 at their first clinic visit were included. Routine demographic and clinical data were collected from clinical correspondence and information systems. All referred patients underwent $24 \mathrm{~h}$ blood pressure monitoring to confirm hypertension. Excel 2010 (Microsoft, Washington, USA) and STATA SE14.2 (StatCorp, Texas, USA) were used to conduct analyses.

Results: A total of 96 patients were referred, $9.4 \%$ were normotensive or had white coat hypertension and $28.2 \%$ were still undergoing investigation, $17.7 \%$ did not attend for follow-up appointments. The remaining 43 patients underwent investigations to exclude secondary hypertension. In these 43 patients the median age was $26 \mathrm{yrs}$, $46.5 \%$ were male, $90.7 \%$ were white, $18.6 \%$ were smokers. Mean body mass index was 32.7 (95\% CI 30.1-35.2). 16.3\% patients had an identifiable secondary cause of hypertension (Table 1). Renal disease was the commonest finding and only a single endocrine-related cause was found.

Conclusions: Within our population of young adults with hypertension $16 \%$ had an identifiable secondary cause with renal disease being the commonest aetiology. This finding is in keeping with similar analyses in other westernised nations [1,2]. Lifestyle factors contribute to many cases of hypertension in young adults and non-attendance at follow-up clinic appointments may hamper long-term management and health promotion.

Disclosures: None

\section{References:}

(1) Viera AJ and Neutze DM, Diagnosis of Secondary Hypertension: An Age-Based Approach, American Family Physician, 2010, 82 (12), 1471-1478.

(2) Van der Merwe W, Hypertension in Young Adults, The New Zealand Medical Journal, 2015, 128 (1409), 75-77.

Table 1 [P - 06]. Causes of secondary hypertension in the cohort

\begin{tabular}{ll}
\hline Aetiology & $\begin{array}{l}\text { Number of } \\
\text { patients }\end{array}$ \\
\hline
\end{tabular}

Chronic Kidney Disease (all cases of reflux 3

nephropathy)

Fibromuscular dysplasia $\quad 1$

Primary hyperaldosteronism 1

Gestational hypertension $\quad 1$

Medication induced hypertension (oral 1 contraceptive pill)

\section{P - 07}

\section{Pre-pregnancy Advice in a Hypertension Clinic}

\section{Marzina Ahmed', Thomas Hinton', Johanna Trinder ${ }^{3}$,} Amy Burchell', Emma Hart ${ }^{4}$, Angus K Nightingale ${ }^{2}$

${ }^{1}$ Bristol Medical School, University of Bristol, United Kingdom, ${ }^{2}$ Department of Cardiology, Bristol Heart Institute, University Hospitals Bristol NHS Foundation Trust, United Kingdom, ${ }^{3}$ Department of Maternal Medicine, University Hospitals Bristol NHS Foundation Trust, United

Kingdom, ${ }^{4}$ School of Physiology, Pharmacology and Neurosciences, University of Bristol, United Kingdom

Introduction: NICE hypertension guidelines [1] recommend angiotensin converting enzyme inhibitors (ACEi) and angiotensin receptor blockers (ARB) as first-line BP treatment for those aged $<55$ years, but these are contraindicated in pregnancy due to risk of teratogenicity and/or fetal renal damage. It is recommended that all females with childbearing potential be counselled about the risks and benefits when using these medications and to consider alternatives when pregnant/planning to conceive.

Methods: We performed an audit of hypertensive females who attended our Specialist Hypertension Clinic 
between January 2015 and June 2017. Clinic letters were reviewed for medications and documentation of prepregnancy counselling.

Results: Of 417 patients ( $45 \%$ female) seen in our Hypertension Clinic, 58 were females aged 18-40 years old, 36 of these were prescribed antihypertensive medication: 23 ACEi, 7 ARB, 6 calcium channel blocker, 1 diuretic and 1 beta blocker; most prescribed by the GP. Half had been pregnant previously. 4 were diabetic and 17 had a BMI over 30. 20/36 (56\%) patients had pre-pregnancy advice documented in their clinic letters.

Conclusions: Pre-pregnancy counselling is important for women with hypertension due to increased risk of complications during pregnancy. In order to improve low rates of documented advice, we suggest that an information sheet is given to all females of child-bearing potential taking antihypertensive medication explaining what to do with their medication if they plan to conceive or become pregnant. Optimal treatment of BP prior to pregnancy remains important in this group to improve long term outcomes.

Disclosures: None

\section{References:}

National Institute for Health and Care Excellence (NICE). Hypertension in Adults: Diagnosis and Management. NICE Guideline (CG127), 2016.

\section{$P-08$ \\ Blood pressure control in non-diabetic patients with early stage CKD. Are we undertreating hypertension?}

\section{Anna M Price ${ }^{1}$, Helen Wheeldon ${ }^{2}$, Lawrence Hopkins ${ }^{1}$, Manvir K Hayer ${ }^{1,2}$, Nicola C Edwards ${ }^{1,2}$, Charles J Ferro $^{1,2}$, Jonathan $N$ Townend ${ }^{1,2}$}

${ }^{1}$ Queen Elizabeth Hospital Birmingham, United Kingdom, ${ }^{2}$ University of Birmingham, United Kingdom

Introduction: Uncontrolled hypertension is a wellknown major modifiable risk factor for both progressive renal disease and cardiovascular risk. Furthermore, prolonged and masked hypertension in chronic kidney disease (CKD) has been associated with left ventricular hypertrophy, adverse cardiac events and proteinuria.

Methods: Non-diabetic patients with early CKD attending routine renal follow up were reviewed against current NICE guidance. Data was collated retrospectively from hospital electronic systems including: eGFR, aetiology, albumin:creatine ratio (ACR), clinic blood pressure, electrolytes and medication. Clinic blood pressures were then compared with $24 \mathrm{~h}$ ambulatory (ABPM) readings.

Results: All patients with an ACR $>70 \mathrm{mg} / \mathrm{mmol}(n=$ 11), were receiving an angiotensin-converting enzyme inhibitor or angiotensin receptor blocker as were the patients who had both hypertension and an ACR $>30$ $\mathrm{mg} / \mathrm{mmol}(n=10)$. There were 39 patients with valid 24 $\mathrm{h}$ ABPM recordings. Of these, 10 had values below target (overall control: $<130 / 80 \mathrm{mmHg}$, day control: < 135/85 mmHg, night control: $<120 / 70 \mathrm{mmHg}$ ). White coat hypertension was only seen once. Of the 29 patients over target, 10 patients also had unsatisfactory clinic values. The remaining 19 had masked uncontrolled hypertension. There were 17 patients with no nocturnal dip in blood pressure, 7 of which were reverse dippers.

Conclusions: White coat hypertension was rare but masked uncontrolled hypertension was frequent and goes undetected in clinic. Those with proteinuria were managed appropriately. A significant proportion of patients were nondippers which has been associated with increased morality and target organ damage.

Disclosures: None

\section{P - 09 \\ High prevalence of hypertensive diabetic patients with Atrial Fibrillation who are not taking anticoagulants}

$\underline{\text { Yasmin Bashir }^{1}}$, Abdul-majeed Salmasi ${ }^{1,2}$

${ }^{1}$ London North West University Healthcare NHS Trust, London, United Kingdom, ${ }^{2}$ Imperial College London, United Kingdom

Background / Introduction: Atrial fibrillation (AF) is the most common arrhythmia with debilitating sequelae. Both systemic hypertension and diabetes mellitus (DM) increase the risk of thromboembolism and subsequently stroke in patients with AF. Anticoagulants significantly reduce the incidence of stroke in these patients.

Purpose: To investigate the prevalence of hypertensive and/or diabetic patients with AF who are not taking anticoagulation in general medical (GP) practices thus identifying a proportion of patients who are at potential risk of developing stroke.

Methods: The AF register was studied across 8 randomly-selected GP practices in the London borough of Brent. The following variables were recorded: age, gender, race, $\mathrm{CHA}_{2} \mathrm{DS}_{2}-\mathrm{VASc}^{1}$ score, presence of hypertension and DM and whether patients were taking anticoagulants and the reason for not taking anticoagulants. 
Results: There were 352 patients (188 males and 164 females) on the AF register. $63 \%$ of these patients had hypertension, $31 \%$ had DM, and $22 \%$ had both hypertension and DM. The following were not taking anticoagulation: $26 \%$ of patients with hypertension, $28 \%$ of patients with DM as well as $29 \%$ of patients with both DM and hypertension, despite being indicated due to a $\mathrm{CHA}_{2} \mathrm{DS}_{2}-\mathrm{VASc}^{1}$ score of $>2$. In $41 \%$ of these patients, anticoagulation was recorded as'contra-indicated' although $22 \%$ of them had already suffered a stroke or TIA. $73 \%$ of the Afro-Caribbean patients with AF had hypertension but $22 \%$ of them were not anticoagulated.

Conclusion: There is a significant proportion of $\mathrm{AF}$ patients who also have hypertension and/or DM and are not taking anticoagulants, despite having a CHA2DS2VASc ${ }^{1}$ score of $>2$. This puts these patients at risk of ischaemic stroke. GPs need to be made more aware of the high risk of patients with $\mathrm{AF}$ and the need for anticoagulation especially if they had hypertension and/or DM.

Disclosure:

None

References:

1. Chadsvasc.org. 2018. ChA2DS2-Vasc / HAS-BLED / EHRA: Atrial Fibrillation risk score calculator. [ONLINE] Available at: https://www.chadsvasc.org [Accessed 14 July 2018].

\section{P - 10 Omitted}

\section{P - 11 \\ Impact on blood pressure control of urinary antihypertensive drug screening in patients with resistant hypertension}

\section{Tzuen Chour Han ${ }^{1}$, Simon Thomas ${ }^{1}$, Alexander Dyker ${ }^{1}$}

${ }^{1}$ Newcastle Upon Tyne Hospitals, United Kingdom,

${ }^{2}$ Newcastle University Medical School, Newcastle upon

Tyne, United Kingdom

Introduction: Studies have shown high levels of nonadherence to blood pressure lowering therapy. We assessed the impact of urine drug screening in patients with resistant hypertension on subsequent blood pressure control.

Methods: We evaluated the results of urinary antihypertensive drug screening in patients with apparent resistant hypertension, when the investigating physician suspected that non- compliance was contributing to poor control. Results were discussed with screened patients. Blood pressure control achieved at the next and the final clinic visits was compared with that of a historic control group, matched for BP, who did not undergo screening.

Results: A total of 34 patients with above target blood pressure were analysed in each group. Screened patients were shown to have all $(32 \%)$, some $(38 \%)$ or none $(32 \%)$ of their prescribed antihypertensive drugs detected in urine. In the screened and control groups respectively, the numbers achieving target blood pressure were $7(21 \%)$ and 2 $(6 \%, \mathrm{P}=\mathrm{NS})$ at the next clinic visit and $15(44 \%)$ and 5 $(15 \%, \mathrm{P}<0.02)$ at the final clinic visit. Median reductions in $\mathrm{BP}$ were $22.5 \mathrm{mmHg}$ (screened) and $6 \mathrm{mmHg}$ (controls) comparing initial and final visits $(p=0.029)$. BP changes between first and next visits were $14 \mathrm{mmHg}$ v $5 \mathrm{mmHg} \mathrm{p}=$ 0.2345. Mann- Whitney U test.

Conclusions: This observational study suggests that urine drug screening has the potential to improve BP control in patients with resistant hypertension. A randomised study of its effectiveness is warranted.

Disclosures: none

\section{P - 12 \\ Systematic review of the effect of indapamide versus bendroflumethiazide for primary hypertension}

Tatiana V Macfarlane ${ }^{1}$, Filippo Pigazzani ${ }^{1}$, Robert WV Flynn $^{1}$, Tom M Macdonald

${ }^{I}$ Medicines Monitoring Unit (MEMO), School of Medicine, University of Dundee, United Kingdom

Introduction: Bendroflumethiazide and indapamide are thiazide and thiazide-like diuretics used to treat arterial hypertension, but their comparative effectiveness is unclear. The aim was to compare efficacy of bendroflumethiazide with indapamide in primary hypertension. The outcomes were all-cause mortality, major cardiovascular events and blood pressure.

Methods: This study was registered with PROSPERO ${ }^{(1)}$. Two reviewers independently performed literature search and study selection, assessed risk of bias and extracted data from included trials. Where there was disagreement, a third reviewer was consulted. Risk ratio (RR) and mean difference were used in a network meta-analysis.

Results: Three trials satisfied the inclusion criteria, two comparing bendroflumethiazide against placebo and one comparing indapamide with placebo. No statistically significant difference was found between the two drugs for all deaths (RR 0.82; 95\% CI 0.57-1.18), cardiovascular and non-cardiovascular deaths (RR $0.82 ; 95 \%$ CI $0.55-1.20$ and $0.81 ; 95 \%$ CI $0.54-1.22$ ) or all cardiovascular events (RR $0.89 ; 95 \%$ CI $0.67-1.18$ ). However, indapamide performed 
worse for stroke (RR 2.21; 95\% CI 1.19-4.11). Three short follow-up trials directly comparing indapamide and bendroflumethiazide were included in a separate analysis of blood pressure. There was no statistically or clinically significant difference between indapamide and bendroflumethiazide in blood pressure reduction irrespective of follow up times.

Conclusions: This review highlights a lack of studies to answer the review question but also a lack of evidence of superiority of one drug over the other. There is a clear need for new studies directly comparing the effect of these drugs on the outcomes of interest.

Disclosures: None

Reference: 1. registration number CRD42017067109; https://www.crd.york.ac.uk/PROSPERO/display_record. php?RecordID $=67109$

$$
\begin{aligned}
& \text { P - } 13 \text { Omitted } \\
& \text { P - } 14 \text { Omitted } \\
& \text { P - } 15 \text { Omitted } \\
& \text { P - } 16 \text { Omitted }
\end{aligned}
$$

\section{P - 17 \\ Accuracy of automated blood pressure measurement in the presence of atrial fibrillation: systematic review and meta-analysis}

\section{Sinead McDonagh ${ }^{1}$, Richard McManus ${ }^{2}$, Christopher Clark $^{T}$}

${ }^{1}$ Primary Care Research Group, University of Exeter Medical School, United Kingdom, ${ }^{2}$ Nuffield Department of Primary Care Health Sciences, University of Oxford, Unite Kingdom

Introduction: Atrial fibrillation (AF) affects up to $3 \%$ of people and is associated with hypertension. Guidelines caution against use of automated blood pressure (BP) measurement devices with $\mathrm{AF}$, and this is usually an exclusion criterion for device validation protocols, creating uncertainty over their accuracy with AF. Some devices also incorporate $\mathrm{AF}$ detection algorithms. We undertook this review to explore accuracy of oscillometric devices for measuring BP.

Methods: Systematic review and meta-analysis: We searched Medline and Embase to November 2017 for studies including people with $\mathrm{AF}$, comparing $\mathrm{BP}$ measurement with any automated device to standard measurement by mercury sphygmomanometer. Study details were extracted by two reviewers. Mean BP differences from standard were extracted or calculated using correlation coefficients from a previous review.
Results: 507 titles and abstracts were screened; we included 13 studies reporting 14 devices. Two ambulatory monitors ( 3 studies) performed well compared to mercury standard for systolic (mean difference $0.5 \mathrm{mmHg}$ (95\% CI -0.9 to 1.9$) ; \mathrm{I} 2=0 \%)$ but not diastolic $\mathrm{BP}(2.0 \mathrm{mmHg}$ $(-2.8$ to 6.8$) ; \mathrm{I} 2=92 \%, \mathrm{p}<0.001)$. Considerable heterogeneity existed between home or office devices (systolic I2 $=81 \%$, diastolic 94\%; $\mathrm{p}<0.001$ ); mean systolic and diastolic differences ranged from -3.1 to $6.1 /-4.6$.to $9 \mathrm{mmHg}$. Overall, devices with AF detection were no more accurate than those without.

Conclusions: The ambulatory monitors studied appear accurate for systolic BP measurement. Marked heterogeneity between other devices, including those with $\mathrm{AF}$ detection, precluded general conclusions. Therefore devices should be evaluated on individual performance. Home or ambulatory BP monitors require validation in populations with $\mathrm{AF}$.

Disclosures: none

\section{P - 18 \\ Prevalence of postural hypotension across care settings and disease cohorts: A systematic review and meta-analysis}

\section{Sinead McDonagh ${ }^{1}$, Natasha Mejzner ${ }^{1}$, Christopher Clark $^{1}$}

${ }^{1}$ University of Exeter, United Kingdom

Introduction: Postural hypotension $(\mathrm{PH})$, the reduction in blood pressure (BP) when rising from seated or supine positions to standing, is a major risk factor for falls, cognitive impairment and mortality. $\mathrm{PH}$ prevalence differs depending on the definition, population, care setting and measurement method and asymptomatic $\mathrm{PH}$ is not routinely tested for in primary care. We aimed to determine the prevalence of $\mathrm{PH}$ across different care settings and disease.

Methods: Systematic review and meta-analyses. Medline and Embase were searched to July 2017 for crosssectional and cohort studies representative of primary care, community or institutional settings which reported $\mathrm{PH}$ prevalence. Data on PH prevalence, definition, measurement method and study level demographics were extracted by two reviewers.

Results: 1497 studies were identified; 82 met the inclusion criteria. Pooled prevalences for the PH consensus definition were $16 \%$ (95\% CI, 13-19\%) in the community, $18 \%(13-24 \%)$ in primary care and $31 \%$ (15-50\%) in care/nursing homes $(\mathrm{P}=0.14$ between groups). Pooled prevalences of $\mathrm{PH}$ for disease subgroups 
were higher [e.g. 19\% (15-23\%) in hypertension] than groups without long-term conditions [13\% (11-16\%); $\mathrm{P}<0.01]$. $\mathrm{PH}$ prevalence was underestimated when the sit-to-stand test was undertaken and the consensus definition was not employed, particularly in poor quality studies.

Conclusions: These results demonstrate substantial prevalences of $\mathrm{PH}$, markedly among institutional care and chronic disease. The PH measurement method, definition and study quality should be considered carefully when checking for PH. Our findings should help to highlight the importance of testing for $\mathrm{PH}$ across care settings and inform future strategies for its management.

Disclosures: None.

$$
\begin{aligned}
& \text { P - } 19 \text { Omitted } \\
& \text { P - } 20 \text { Omitted }
\end{aligned}
$$

\section{P - 21}

\section{Using a weighted genetic risk core to investigate the clinical impact of genetically determined Systolic Blood Pressure in a Large Prospective Cohort}

\author{
Alexander Doney ${ }^{1}$, Robert Flynn ${ }^{1}$, Tsz Man ${ }^{1}$, Colin \\ Palmer ${ }^{1}$, Louise Donnelly ${ }^{1}$, Steven Morant ${ }^{1}$, Isla \\ Mackenzie $^{1}$, Thomas MacDonald ${ }^{1}$ \\ ${ }^{1}$ University of Dundee, United Kingdom
}

Introduction: We investigated the clinical impact of a 162 variant weighted genetic score (wGS) for systolic blood pressure (SBP) in the type 2 diabetes (T2D) GoDARTS bioresource ${ }^{1}$, linked to long-term electronic medical records.

Methods: The wGS was constructed using published coefficients from genome-wide association studies. Each SBP value was labelled according to the number of antihypertensive therapies the patient was taking at the time of measurement. Mixed linear models for repeated measures were used to investigate the impact of the wGS on SBP, and logistic and Cox's regression to investigate Major Adverse Cardiac Events (MACE).

Results: The study comprised 8,034 patients (97\% with T2D) and 382,305 SBP recordings (median 54 [IQR 41-70]) per individual over a median of 11.6 (IQR 6.9-16.5) years. $8.9 \%$ of SBP variance was explained by wGS, age and sex. Patients were on significantly more antihypertensive therapies $(\mathrm{p}=4.7 \times 10-15)$ in the wGS top tertile compared with the bottom where, after adjustment for therapy, SBPs were higher by $3.1 \mathrm{mmHg}$ (95\%CI $2.4-3.8 ; \mathrm{p}=9.1 \times 10-18)$. Patients in the top
wGS tertile were significantly more likely to have a history of hospitalisation for MACE (OR 1.19, 95\%CI $1.05-1.34 ; \mathrm{p}=0.007)$ and during 10 year follow-up a $25.2 \%(95 \%$ CI $8.6-44 ; p=0.002)$ increased risk of cardiovascular death.

Conclusions: Patients in the top tertile of wGS have higher SBP and remain at increased risk of MACE despite increased antihypertensive therapy. With a rapidly increasing proportion of SBP variability being explained by genetic factors, such instruments may soon be used clinically to improve cardiovascular risk management.

Disclosures: None

Reference:

1.Hébert, H. L. et al. Cohort Profile: Genetics of Diabetes Audit and Research in Tayside Scotland (GoDARTS). Int. J. Epidemiol. (2017).

\section{P - 22}

Blood pressure and hypertension in relation to cognitive performance in older Irish adults from the TUDA cohort: Preliminary analysis

Harry Jarrett ${ }^{1}$, Katie Moore ${ }^{1}$, Catherine Hughes ${ }^{1}$, Kirsty Porter ${ }^{1}$, Leane Hoey ${ }^{1}$, Mary Ward ${ }^{1}$, J Strain ${ }^{1}$, Anne Molloy ${ }^{2}$, Conal Cunningham ${ }^{3}$, Miriam Casey ${ }^{3}$, Eamon Laird $^{2}$, Kevin McCarroll ${ }^{3}$, Kristina Pentieva ${ }^{7}$, Helene McNulty ${ }^{7}$

${ }^{I}$ Nutrition Innovation Centre for Food and Health, Ulster University, Coleraine, Northern Ireland, ${ }^{2}$ Institute of Molecular Medicine, School of Medicine, Trinity College Dublin, Ireland, ${ }^{3}$ Mercers Institute for Research on Ageing, St James's Hospital, Dublin, Ireland

Introduction: By 2050, the number of people aged 60 years and over is projected to reach 2 billion, of which an estimated 131 million will have dementia. There is a need to identify modifiable factors for targeted interventions to promote brain health in ageing populations. The aim of this investigation was to examine blood pressure (BP) and hypertension as determinants of cognition and rate of cognitive decline over a 5-year follow-up period in healthy older adults.

Methods: Baseline data on adults aged 60-67 years $(n$ $=1267$ ) were drawn from the Trinity, Ulster, Department of Agriculture (TUDA) study[1] of older Irish adults. A separate analysis was conducted on a subset of TUDA participants, aged $>68$ years who were reinvestigated five years after their initial sampling $(n=587)$. At both time points, BP was recorded and cognitive function was assessed using the following tests: Mini-Mental State Examination (MMSE), Repeatable Battery for the 
Assessment of Neuropsychological Status (RBANS) and Frontal Assessment Battery (FAB). For this analysis, cognitive dysfunction was defined as a score in the lowest quartile $(25 \%)$ in each test.

Results: Analysis of the baseline TUDA cohort showed that, after adjustment for significant covariates (age, education, depression, alcohol intake and smoking status), neither systolic nor diastolic BP was a significant determinant of cognitive dysfunction. However, when component domains of the RBANS were examined, hypertension was associated with a significantly higher the risk of cognitive dysfunction in the Language domain (OR: 1.404, 95\% CI: 1.01-1.96 $\mathrm{p}<0.05)$. At the 5-year follow-up, a significant decline in all three cognitive tests was observed $(\mathrm{p}<0.001)$, but neither BP nor hypertension was found to be a significant predictor of the rate of cognitive decline.

Conclusions: These preliminary findings suggest that hypertension may have an adverse influence on certain cognitive domains in ageing but this requires confirmation.

Disclosures: None

Reference:

[1] McCarroll K, Beirne A, Casey M, et al. Determinants of 25-hydroxyvitamin D in older Irish adults. Age Ageing 2015; 44:847-853; https://www.ncbi.nlm.nih.gov/pubmed/ 26265675

\section{P - 23 \\ Prospective external validation of the Predicting Out-of-OFfice Blood Pressure (PROOF-BP) strategy for triaging ambulatory monitoring in the diagnosis and management of hypertension: an observational cohort study}

\section{James P Sheppard ${ }^{11}$, Richard Stevens', Una Martin ${ }^{2}$, Jonathan Mant ${ }^{3}$, Richard Hobbs ${ }^{1}$, Richard McManus ${ }^{1}$ \\ ${ }^{1}$ University of Oxford, United Kingdom, ${ }^{2}$ University of Birmingham, United Kingdom, ${ }^{3}$ University of Cambridge, United Kingdom}

Introduction: Triaging patients with suspected high blood pressure for ambulatory blood pressure monitoring (ABPM), on the basis of their clinic blood pressure and characteristics, may reduce the need for ABPM. This study aimed to prospectively validate this new approach using data from routine practice.

Methods: Consecutive patients aged $>18$ years, referred for ABPM were enrolled from ten primary care practices and one hospital. All participants underwent ABPM and had the new triage algorithm (PROOF-BP) applied. The primary outcome was the proportion of patients correctly classified with hypertension using the triaging strategy, compared to the reference standard of daytime ABPM.

Results: A total of 887 patients (mean age $52.8 \pm 16.2$ years) were referred ABPM and enrolled during the study period. The triaging strategy correctly classified hypertensive status in 801 patients $(90 \%, 95 \% \mathrm{CI} 88 \%-92 \%)$ and had a sensitivity of $97 \%$ (95\% CI $96 \%-99 \%)$ and specificity of $76 \%$ (95\% CI $71 \%-81 \%$ ) for hypertension. Use of triaging, rather than uniform referral for ABPM would have resulted in 435 patients (49\%, 95\% CI 46\%-52\%) being referred for ABPM and the remainder managed on the basis of their clinic measurements. Of these, $69(8 \%, 95 \%$ CI 6\%-10\%) would have received treatment that would have been deemed unnecessary had they received ABPM.

Conclusions: In a population of patients referred for ABPM, this new triaging approach accurately classified hypertensive status for most, with half the utilisation of ABPM compared with usual care. This triaging strategy can therefore be recommended for diagnosis or management of hypertension in patients where ABPM is being considered.

Disclosures: None

\section{P - 24 \\ Characteristics of Americans with Stage 1 Hypertension: United States National Health Nutrition and Examination Survey (NHANES) 2011-2016}

\section{Yue Fei ${ }^{1}$, Man Fung Tsoi ${ }^{1}$, Tommy Tsang Cheung ${ }^{1}$, Bernard Man Yung Cheung ${ }^{1}$}

${ }^{1}$ University of Hong Kong, Hong Kong

Introduction: Recent American guidelines defined stage 1 hypertension as a systolic blood pressure (BP) of $130-139 \mathrm{mmHg}$ or a diastolic BP of $80-89 \mathrm{mmHg}$ [1]. We therefore analysed the characteristics of people with stage 1 hypertension.

Methods: Participants of NHANES 2011-2016 with BP measurements and not on antihypertensive medication were included in this analysis. Abdominal obesity was defined as a waist circumference $\geq 102 \mathrm{~cm}$ in men and $\geq 88 \mathrm{~cm}$ in women. Results were analysed using $\mathrm{R}$ statistics version 3.3.3 with statistical package "survey" version 3.32-1.

Results: Among 8,154 hypertensive participants, 1,818 adults $(22 \%)$ had stage 1 hypertension. Mean $\mathrm{BP} \pm \mathrm{SD}$ was $126.5 \pm 8.2, \quad 129.1 \pm 7.2, \quad$ and $129.0 \pm 6.9 \mathrm{mmHg}$ in 2011-2012, 2013-2014, 2015-2016, respectively. 80.4 (95\% CI: 75.7-84.5)\%, 77.4 (70.6-83.3)\%, and 80.8 (75.6-85.4)\%, respectively, were below the age of 60 and 39.8 (34.6-45.2)\%, $41.2(35.8-46.6) \%$ and $44.0(40.0-48.1) \%$ were female. $57.4(48.4-66.0) \%, 63.6(58.9-68.1) \%$, and 64.0 
(56.1-74.5)\% had abdominal obesity. Only $2.7 \%$ $(1.4 \%-4.6 \%), 3.9 \%(1.9 \%-6.9 \%)$ and $2.2 \%(1.4 \%-3.2 \%)$ had a previous history of cardiovascular disease.

Conclusions: Most people with stage 1 hypertension are obese men and women under the age of 60 years. Few have a history of cardiovascular disease yet, so they would all benefit from lifestyle intervention to address risk factors including but not limited to hypertension.

Disclosures: None.

Reference:

1. Whelton PK, et al. J Am Coll Cardiol 2017. https:// doi.org/10.1016/j.jacc.2017.11.006

\section{P - 25}

\section{The heart-brain connection in the 1946 British Birth Cohort}

Chloe Park ${ }^{1}$, Marcus Richards ${ }^{2}$, Rebecca Hardy ${ }^{2}$, Nish Chaturvedi $^{1}, 2$, Alun Hughes ${ }^{1}$

${ }^{1}$ Institute of Cardiovascular Science, UCL, London, United Kingdom, ${ }^{2}$ MRC Unit for Lifelong Health and Ageing, UCL, United Kingdom

Introduction: Recent studies have reported a strong association between cardiovascular disease and cognitive impairment. However, our understanding of the relationship remains incomplete and determining if the association is causal remains problematic.

Methods: 1490 individuals (60-64years) underwent echocardiography for systolic, diastolic and global Left Ventricle (LV) function. Cognitive function was assessed aged 8 yrs, 60-64 yrs and 69 yrs. Multivariable regression analyses assessed the associations of LV function with cognitive function at these ages.

Results: Poorer LV diastolic function was significantly associated with lower scores in verbal memory $(\beta \pm \mathrm{SE}:-0.26 \pm 0.08, \quad \mathrm{P}<0.0001)$ and letter search speed $\quad(-0.28 \pm 0.08, \quad \mathrm{P}<0.0001)$ aged $60-64$ years. Poorer LV diastolic function was also associated with poorer scores in memory $(-0.30 \pm 0.08, \mathrm{p}<0.0001)$, letter search speed $(-0.23 \pm 0.08, \mathrm{p}=0.006)$, verbal fluency $(-0.31 \pm 0.08, \mathrm{p}<0.0001)$, visuospatial abilities $(-0.26 \pm 0.08, \mathrm{p}=0.002)$ and total cognition $(-0.29 \pm$ $0.08, \mathrm{p}=0.001$ ) aged 69years. Adjustment for education, cardiometabolic and vascular risk factors (blood pressure, macro/microvascular disease) attenuated these associations. Higher childhood cognition was associated with better $\mathrm{LV}$ diastolic function age 60-64years $(-0.052 \pm 0.1 \mathrm{~cm} 2, \mathrm{p}<0.0001)$. This association was reduced but remained after adjustment for highest educational achievement and cardiometabolic risk factors at the time of the echocardiogram and vascular pathways.

Conclusions: LV dysfunction and cognitive ability are associated across a range of ages. While associations in mid-to-later life appear to be confounded by common causes, the association between childhood cognition and future LV diastolic function remain unexplained. These results imply that childhood cognitive function has an independent association with $\mathrm{LV}$ function in later life and could provide further insight into the causal direction of the heart-brain connection.

Disclosures: None.

\section{P - 26 \\ Hypertension control and 5-year arterial stiffness progression}

\section{Carlos Valencia-Hernandez ${ }^{1}$, Sara Ahmadi-Abhari ${ }^{1}$, Martin J. Shipley ', Carmel McEniery', lan Wilkinson",} Eric J. Brunner

${ }^{1}$ Research Department of Epidemiology and Public Health, University College London, United Kingdom, ${ }^{2}$ Division of Experimental Medicine and Immunotherapeutics, University of Cambridge, United Kingdom

Introduction: Arterial stiffness is a surrogate measure of cardiovascular ageing, associated with higher risk of cardiovascular events and mortality [1]. The aim of this report was to estimate the differences in the progression of arterial stiffness according to hypertension control in a cohort of older individuals.

Methods: The Whitehall II study is an on-going cohort of civil servants followed every 4-5 years. Data used for this analysis consists of two measurements of pulse wave velocity (PWV) and blood pressure separated by 5 years taken during phases 9 (2008-2009) and 11 (2012-2013), from 4998 participants (3680 men; 1318 women) [2]. Blood pressure control was categorized according to hypertension status and hypertension medication. Cross-sectional and 5year change models were fitted using linear mixed model techniques.

Results: There were 1842 (36.9\%) controlled hypertensive, $871(17.4 \%)$ untreated hypertensive and 557 (11.1 $\%)$ uncontrolled hypertensive participants in the total sample. A model adjusted for sociodemographic characteristics, comorbidities and health behaviours showed that compared to non-hypertensives, mean PWV increase was $0.04 \mathrm{~m} / \mathrm{s}$ (95\% CI: $-0.04,0.17 \mathrm{p}: 0.51$ ) for controlled hypertensives, $0.20 \mathrm{~m} / \mathrm{s} \quad(95 \% \mathrm{CI}: \quad 0.06,0.35 \quad \mathrm{p}<0.001) \quad$ untreated 
hypertensives and 0.25 (95\%CI: $0.03,0.47 \mathrm{p}<0.05$ ) for uncontrolled hypertensives.

Conclusions: Our findings suggest that untreated and poor controlled hypertension is associated with higher aortic stiffness at baseline and higher progression rate of aortic stiffness than normal blood pressure over a 5-year period. PWV assessment could be a more accurate indicator of the chronic impact of hypertension treatment, unbiased to the instantaneous levels of blood pressure.

Disclosures: none

References:

1. Ben-Shlomo Y, Spears M, Boustred C, May M, Anderson SG, Benjamin EJ, et al. Aortic pulse wave velocity improves cardiovascular event prediction: an individual participant meta-analysis of prospective observational data from 17,635 subjects. J Am Coll Cardiol [Internet]. 2014 Feb 25 [cited 2016 Apr 1];63(7):636-46. Available from: http://content.onlinejacc.org/article.aspx? articleid $=1767250$

2. Marmot M, Brunner E. Cohort Profile: The Whitehall II study. Int J Epidemiol [Internet]. 2005 Apr 1 [cited 2018 Aug 14];34(2):251-6. Available from: http://academic.oup. com/ije/article/34/2/251/746997/Cohort-Profile-The-Whitehall-II-study

\section{P - 27}

\section{Symptoms of orthostatic intolerance and cerebral perfusion-Data from The Irish Longitudinal Study on Ageing (TILDA)}

\section{Triona McNicholas ${ }^{1}$, Robert Briggs ${ }^{1}$, Paul Claffey ${ }^{2}$, Louise Newman ${ }^{1}$, Katy Tobin ${ }^{1,3}$, Rose Anne Kenny ${ }^{1,2}$ \\ ${ }^{1}$ The Irish Longitudinal Study on Ageing, Trinity College Dublin, Ireland, ${ }^{2}$ Department of Medical Gerontology,}

Trinity College Dublin, Ireland, ${ }^{3}$ Global Brain Health Institute, Trinity College Dublin, Ireland

Introduction: Orthostatic intolerance (OI), symptoms such as light-headedness after standing, is often observed in orthostatic hypotension $(\mathrm{OH})$ but can also be observed in people with normal orthostatic cardiovascular haemodynamics. This study assesses cerebral perfusion during orthostasis in individuals with OI, both with and without $\mathrm{OH}$, in a large cohort of community-dwelling adults.

Methods: Beat-to-beat BP was measured during active stand. $\mathrm{OH}$ was defined as a drop $\geq 20 \mathrm{mmHg}$ of systolic $\mathrm{BP}$ or $\geq 10 \mathrm{mmHg}$ diastolic BP at $40 \mathrm{~s}$ post stand. In parallel, a continuous index of frontal lobe perfusion, Tissue Saturation Index (TSI) was measured using near-infrared spectroscopy (NIRS). Participants were asked about orthostatic symptoms to define OI. Linear regression assessed whether baseline TSI and nadir TSI were different between groups. Mixed-effects regression compared TSI at each time point post stand.

Results: 2884 participants were included, $6.6 \%$ of whom had OI. 5.65\% of participants had OI without OH. Baseline $(\beta-0.91 ; 95 \% \mathrm{CI}-1.60,-0.17$; $\mathrm{p}$-value 0.016$)$ and nadir $(\beta-0.85 ; 95 \% \mathrm{CI}-1.62,-0.07$; $\mathrm{p}$-value 0.032$)$ TSI were lower in those with OI. Categorising by $\mathrm{OH}$ status, participants with OI without $\mathrm{OH}$ had lower baseline TSI $(\beta$ $-0.89 ; 95 \%$ CI $-1.69,-0.10$; p-value 0.029 ), and displayed a trend towards lower nadir TSI, $(\beta-0.80 ; 95 \% \mathrm{CI}$ $-1.64,0.40$; $p$-value 0.062 ). TSI at each time point is displayed graphically (Fig. 1).

Conclusions: In a large cohort of older adults, orthostatic symptoms after standing were associated with a lower index of frontal lobe perfusion, not mediated by orthostatic BP drop.

Disclosures: None

$\mathrm{OH}$ - Orthostatic hypotension, OI - Orthostatic intolerance

\section{P - 28 Omitted}

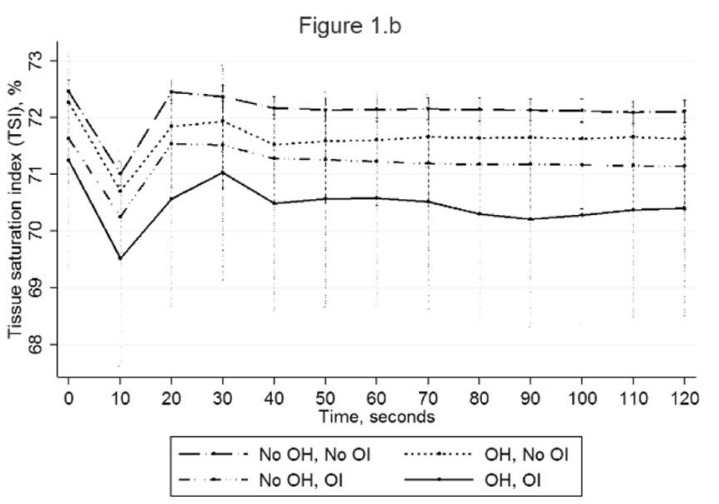

Fig. 1 [P - 27]. Cerebral perfusion during stand by OI status (1.a) and cerebral perfusion by OH/OI status (1.b) 


\section{P - 29}

\section{Sex differences in cerebral haemodynamics during active stand in an older population}

\author{
Louise Newman ${ }^{1}$, Dan Carey ${ }^{1}$, Rose Anne Kenny ${ }^{1}$ \\ ${ }^{I}$ Trinity College Dublin, Ireland
}

Introduction: Hypotension due to orthostatic stress has been associated with mortality and cardiovascular events in middle-age. Here we use near infra-red spectroscopy (NIRS) to examine cerebral haemodynamics during an orthostatic challenge. We investigate the influences of sex and age on cerebral oxygenation during an active stand in an older population.

Methods: Cross-sectional data $(n=3,120)$ from The Irish Longitudinal Study on Ageing (TILDA), the nationally representative sample of adults aged 50 and over was used. Participants rested in the supine position for $\sim 10 \mathrm{~min}$ before standing and remained standing for $180 \mathrm{~min}$. Absolute cerebral oxygenation was measured continuously measured from the frontal lobe via a non-invasive single channel NIRS. Continuous beat-to-beat blood pressure was measured simultaneously. Multi-level mixed models were used to examine changes in cerebral perfusion over time. Models were adjusted for age, sex, education and systolic blood pressure.

Results: There were no sex differences in absolute baseline cerebral oxygenation. However, males exhibited a larger absolute drop $(-1.70, \mathrm{CI}:-1.83-1.57$ vs $-1.00, \mathrm{CI}$ : $-1.12-0.88)$ and $\%$ change $(-2.32$, CI: $-2.50-2.15$ vs -1.40 , CI: $-1.56-1.24)$ from baseline in cerebral oxygenation after standing than females. Females exhibited a different pattern of recovery, and remained lower than males after $180 \mathrm{~s}$.

Conclusions: We have shown in a large population that the impact of orthostatic stress on the cerebral oxygenation of males and females is very different and may put females at greater risk of cerebral hypoperfusion.

Disclosures: none

\section{P - 30}

Left ventricular hypertrophy in hypertensive heart disease: early myocardial response to treatment

\author{
Andrew Jordan ${ }^{1}$, Christine Anning ${ }^{2}$, Claire Ball ${ }^{2}$, Nicola \\ Pamphilon $^{2}$, Lindsay Wilkes ${ }^{2}$, Jon Fulford ${ }^{2}$, Angela \\ Shore $^{1,2}$, Andrew Sharp ${ }^{1,3}$, Nicholas Bellenger ${ }^{1,3}$ \\ ${ }^{1}$ University of Exeter, United Kingdom, ${ }^{2}$ NIHR Exeter \\ Clinical Research Facility, United Kingdom, ${ }^{3}$ Royal Devon
}

and Exeter Hospital NHS Foundation Trust, United Kingdom

Introduction: Left ventricular hypertrophy (LVH) is an independent predictor of adverse outcomes and associated with reduced longitudinal and radial myocardial strain (deformation per unit length), and increased torsion. This study assessed whether changes in cardiac structure and function occur after only 18 weeks' antihypertensive treatment.

Methods: 55 patients (18-79 years) with never-treated grade 2 hypertension were enrolled in an 18-week protocoldriven programme, undergoing treatment intensification every 2-4 weeks to achieve office BP target. MRI (1.5 T) LV volumes, mass, strain and torsion were measured at enrolment and 18 weeks using commercially-available processing software (Extended MR Workspace, Philips Healthcare) and feature tracking analysis (TomTec Imaging Systems) respectively.

Results: $41 / 55$ participants had MRI at both visits, their mean office BP reduced from $174 \pm 16 / 103 \pm 9 \mathrm{mmHg}$ to $132 \pm 12 / 80 \pm 8 \mathrm{mmHg}$. LV mass reduced from $89.0 \pm 3.8$ to $76.8 \pm 3.4 \mathrm{~g}, \mathrm{p}<0.0001$, left ventricular ejection fraction reduced from $65.6 \pm 1.1$ to $63.4 \pm 1.1 \%, \mathrm{p}=0.03$, accompanied by a significant reduction in short axis radial strain $(48.6 \pm 18.2$ to $41.7 \pm 19.7, \mathrm{p}<0.0001)$, mid-ventricular circumferential strain $(-20.8 \pm 4.9$ to $-19.1 \pm 3.7, \mathrm{p}=$ $0.023)$ and apical circumferential strain $(-26.0 \pm 5.3$ to $-23.4 \pm 4.2, \mathrm{p}=0.003$ ).

Conclusions: We show for the first time that LVH regresses rapidly and myocardial function is altered after only 18 weeks' antihypertensive treatment in treatmentnaïve patients with grade 2 hypertension.

Disclosures: none

\section{P - 31 \\ Evaluating Diuretics in Usual Care (EVIDENCE)- piloting a novel methodology for comparative effectiveness research in hypertension}

\section{Amy Rogers ${ }^{1}$, Alexander S F Doney ${ }^{1}$, Angela Flynn ${ }^{1}$, Robert W V Flynn ${ }^{1}$, Isla S Mackenzie ${ }^{1}$, Thomas M MacDonald $^{1}$ \\ ${ }^{I}$ MEMO Research, University of Dundee, United Kingdom}

Introduction: In 2011, NICE recommended thiazide-like diuretics be used in preference to thiazide diuretics to treat hypertension. However, there was acknowledgement that this guidance was criticised by some as lacking sufficient evidential basis. The Evaluating Diuretics in Normal Care (EVIDENCE) study uses cluster randomisation of 
prescribing policy to compare indapamide (thiazide-like) with bendroflumethiazide (thiazide). EVIDENCE exploits existing routine NHS activity and data collection to efficiently compare safety and cardiovascular outcomes. The pilot study will explore the feasibility of this approach in up to 30 practices.

Methods: All patients prescribed repeat bendroflumethiazide or indapamide in participating General Practices are identified and those unsuitable for a drug switch for clinical reasons excluded. Patients are informed of the prescribing evaluation taking place and the potential for a prescription change. Randomisation is then applied at practice level and existing prescriptions switched, where necessary, using existing mechanisms, to comply with randomized policy. Routinely collected NHS data will be used to link prescribing to deaths/hospitalisations data for analysis.

Results: Eleven practices have so far been randomised in Tayside (mean list size 6306, range 2062-10744). The mean number of patients identified in each practice is 272 (101-570). In all practices, bendroflumethiazide was the dominant drug before randomisation, taken by $79 \%$ of patients (64-94\%). 77 individuals were excluded from switching (0-17 per practice) and only 11 refused a medication switch.

Conclusions: The EVIDENCE methodology will efficiently compare the effectiveness of two antihypertensive medications.

Disclosures: EVIDENCE is supported by the BIHS Collaborative Research Working Party.

\section{P - 32 Omitted}

\section{P - 33}

Treatment of white coat Hypertension in the Very Elderly Trial (HYVET 2)-A UK feasibility study for a randomised controlled trial

\author{
Michael Okorie ${ }^{1}$, Khalid Ali ${ }^{1}$, Stephen Bremner ${ }^{1}$, \\ Richard Quirk', Stephen Jackson ${ }^{3}$, John Potter ${ }^{4}$, Nigel \\ Beckett $^{5}$, Christopher Bulpitt ${ }^{6}$, Chakravarthi Rajkumar ${ }^{1}$ \\ ${ }^{1}$ Brighton And Sussex Medical School, United Kingdom, \\ ${ }^{2}$ Sussex Community NHS Foundation Trust, Brighton, \\ United Kingdom, ${ }^{3}$ Kings College London, United Kingdom, \\ ${ }^{4}$ University of East Anglia, Norwich, United Kingdom, \\ ${ }^{5}$ Guy's and St Thomas' NHS Foundation Trust, London, \\ United Kingdom, ${ }^{6}$ Imperial College London, United \\ Kingdom
}

Introduction: The treatment of hypertension in older people was controversial until results from the Hypertension in the Very Elderly Trial (HYVET) demonstrated benefit in those 80 years or older. The HYVET ambulatory blood pressure (BP) sub study analysis of a small representative sample reported up to $60 \%$ of patients in the main study may have had white coat hypertension (WCH)[1], defined as clinic BP readings $\geq 140 / 90 \mathrm{mmHg}$ and ambulatory BP readings $<135 / 85 \mathrm{mmHg}$. Currently treatment is not recommended for such individuals.

This study serves as a prelude to a definitive randomised controlled trial (HYVET 2) to determine whether the treatment of WCH in the very elderly is feasible and outweighs any adverse events.

Methods: Open-label study assessing the feasibility of a randomised controlled trial to treat $\mathrm{WCH}$ in primary care in 100 patients aged $\geq 75$ years with equal randomisation to a treatment arm (indapamide and perindopril) and control arm (no treatment). Recruitment from May 2018; 52 weeks' follow up.

Results: Will report patient recruitment, adherence and withdrawal rates, willingness of GPs to recruit and randomise patients and the frequency of a composite of cardiovascular events.

Conclusions: It is unknown whether older adults with WCH benefit from treatment. This feasibility study will inform the planning of a definitive trial in an attempt to address this research question.

Key words: White Coat Hypertension, Elderly

Disclosures: Study funded by a research grant from the Dunhill Medical Trust. Authors declare no competing interests.

\section{References:}

1. Bulpitt CJ et al. Hypertension. 2013 Jan;61(1):89-94. https://doi.org/10.1161/HYPERTENSIONAHA.112. 191791. Epub 2012 Nov 19. PMID: 23172934

\section{P - 34 \\ Home blood pressure monitors owned by participants in the Treatment In Morning versus Evening (TIME) study}

\section{Thineskrishna Anbarasan ${ }^{1}$, Amy Rogers ${ }^{1}$, David Rorie ${ }^{1}$, Kerr Grieve', Thomas MacDonald ${ }^{1}$, Isla Mackenzie ${ }^{1}$ \\ ${ }^{1}$ Medicines Monitoring Unit (MEMO) and Hypertension Research Centre, University of Dundee, United Kingdom}

Introduction: Self-monitoring with home blood pressure monitors (HBPMs) can be an effective strategy to improve blood pressure (BP) control ${ }^{(1)}$. Various HBPMs are available for purchase but only some are validated. This study aimed to assess whether HBPMs owned by patients taking part in a clinical trial were validated models. 
Methods: The TIME study ${ }^{(2)}$ is a randomised trial investigating the effect of antihypertensive medication dosing time on cardiovascular outcomes in participants with hypertension ${ }^{(3)}$. We identified the model of HBPM reported by participants, then cross-referenced this against lists of validated HBPMs produced by dabl Educational Trust ${ }^{(4)}$ and the British and Irish Hypertension Society (BIHS) ${ }^{(5)}$.

Results: Of 21,114 participants, 10,464 (49.6\%) reported owning a HBPM. 7,485 of these (71.5\%) participants owned a monitor that could be identified. Of these, 5,962 (79.7\%) participants owned a validated monitor, either by dabl $(n=5,877)$ or BIHS $(n=5,395)$. $1,523(20.3 \%)$ participants owned an identifiable HBPM lacking clear evidence of validation. 6,984 (93.3\%) and 501 (6.7\%) participants owned an upper arm and wrist HBPM respectively. Validated HBPMs had a higher median online retail price of $£ 45.00$ compared to $£ 20.00$ for HBPMs lacking clear evidence of validation.

Conclusions: This study outlines the types of HBPMs owned by people with hypertension in the UK. A significant number of participants own HBPMs lacking evidence of validation and the effect of this on the reliability of selfreported BP measurements remains unclear. The public may benefit from clearer information about validation status of HBPMs to facilitate informed purchases.

Disclosures: The TIME study is funded by the British Heart Foundation.

\section{References:}

1.McManus RJ, Mant J, Franssen M, Nickless A, Schwartz C, Hodgkinson J, et al. Efficacy of self-monitored blood pressure, with or without telemonitoring, for titration of antihypertensive medication (TASMINH4): an unmasked randomised controlled trial. Lancet. 2018; 391: 949-59

2. TIME study registration numbers: EudraCT 2011001968-21, ISRCTN18157641

3.Rorie D, Rogers A, Mackenzie I, et al. Methods of a large prospective, randomised, open-label, blinded endpoint study comparing morning versus evening dosing in hypertensive patients: the Treatment In Morning versus Evening (TIME) study. BMJ Open. 2016;6(2)

4.dabl Educational TrustlMonitors for Self-measurement of Blood Pressure (SBPM) [Internet]. Dableducational.org. 2018 [cited 29 March 2018]. Available from: http://www.da bleducational.org/sphygmomanometers/devices_2_sbpm. html\#ArmTable

5.For Home Use-British and Irish Hypertension Society I Registered UK Charity No. 287635 [Internet]. British and Irish Hypertension Society I Registered UK
Charity No. 287635. 2018 [cited 29 March 2018]. Available from: https://bihsoc.org/bp-monitors/for-home-use/

\section{$P$ - 35 \\ Using Telehealth to Remotely Adjust Anti- Hypertensive Regimes and Reduce Hospital Clinic Appointments}

\section{Gwyneth Lawson ${ }^{1}$, Madhavan Menon ${ }^{1}$, Richard Fish ${ }^{1}$}

${ }^{1}$ University Hospital of North Midlands NHS Trust, Stoke On Trent, United Kingdom

Introduction: All patients referred to our hypertension clinic who self-measure their blood pressure (BP) are offered the use of a free mobile texting service (Florence or 'FLO'). For patients requiring aldosterone:renin ratio (ARR) measurement, our current practice is to switch antihypertensive medications prior to testing to those that do not interfere with the assay. We investigated the utility of FLO for remotely monitoring BP following medication alterations in this context with particular emphasis on whether clinic appointments could be reduced.

Methods: FLO was offered to all patients undergoing changes to medication prior to ARR measurement. Following a change in medication or dose, patients were asked to monitor BP for 4-7 days and to send in readings via FLO. These were then reviewed and acted upon as necessary.

Results: During 2017, 158 patients were offered FLO. 92 completed registration. 41 patients required ARR testing following registration and we received BP readings for 21. Of these, 15 had medications adjusted. 5 others preferred to send their BP readings by email despite being registered for FLO. Following ARR testing 37 patients using FLO were converted back to 'first-line' medications without the need for a clinic visit. We made changes to medication for 6 patients who did not attend clinic based upon BP readings sent through FLO.

Conclusion: FLO has been useful in managing patients undergoing temporary medication changes for the purpose of ARR testing. Particular advantages include reducing clinic appointments thereby improving patient experience and improving cost effectiveness of our service.

Disclosure: None 


\section{P - 36}

Pre-load reduction decreases myocardial wall stress despite a small impact on blood pressure: a potential strategy to revert cardiac remodelling?

\section{Luca Faconti ${ }^{1}$, Haotian Gu$^{1}$, Bushra Farukh ${ }^{1}$, Phil Chowienczyk $^{1}$}

${ }^{1}$ King's College London, United Kingdom

Introduction: Increased myocardial wall stress (MWS) promotes left ventricular (LV) hypertrophy (LVH) in hypertensive (HT) patients. LVH regression with antihypertensive drugs has been ascribed to reduction in blood pressure (BP) and/or pharmacological properties but it might also be explained by hemodynamic actions on MWS. Here, we examined the effects of nonpharmacological pre-load reduction on BP and MWS in HT subjects.

Methods: HT patients had radial applanation tonometry (SphygmoCor AtCor Medical, Sydney, Australia) calibrated by the average of 3 brachial BP and trans-thoracic echocardiography (TTE) to obtain LV pressure and volume curves from which MWS was calculated. All measurements were repeated after non-invasive pre-load reduction by pneumatic lower limbs venous occlusion (VO). Inferior vena cava diameter (IVCd) by TTE was used to assess change in cardiac pre-load.

Results: In 69 HT subjects, age (mean \pm SD) $44.7 \pm 14.3$ years, BP $140.9 \pm 17.5 / 89.1 \pm 13.3 \mathrm{mmHg}, 32 \%$ with $\mathrm{LVH}$, VO decreased IVCd (mean, $95 \%$ confidence interval) by 0.3 $(-0.3,-0.2) \mathrm{cm}$ and $\mathrm{LV}$ end systolic volume by $7.7(-9.5$, $-5.9) \mathrm{ml}$, both $\mathrm{P}<0.01$.

Diastolic BP and heart rate did not change significantly while systolic BP decreased by $3.0(-4.6,-1.4) \mathrm{mmHg}, \mathrm{P}<$ 0.01. After VO, both peak MWS and mean MWS were reduced: $-56.1(-82.3,-29.7) \mathrm{kdynes} / \mathrm{cm} 2$ and -49.7 $(-72.1,-27.5)$ kdynes/cm2, respectively, both $\mathrm{P}<0.01$. This $\approx 12 \%$ decrease for peak and mean MWS was achieved with a small change in $\mathrm{BP}(\approx 2 \%)$.

Conclusions: Reduction of cardiac pre-load decreases MWS despite a small effect on BP. Sustained pre-load reduction in HT could be a strategy to prevent/revert cardiac remodelling.

Disclosures: None
P - 37

Reducing sympathetic activity and cardiac pre-load have differential effects on mean and pulsatile blood pressure components

\author{
$\underline{\text { Luca Faconti }^{1}}$, Bushra Farukh ${ }^{1}$, Phil Chowienczyk ${ }^{1}$ \\ ${ }^{1}$ King's College London, United Kingdom
}

Introduction: Blood pressure (BP) can be divided into steady state (mean arterial pressure, MAP) and pulsatile (pulse pressure, PP) components that are differently associated with target organ damage and cardiovascular events independently of brachial BP. Here we tested the effects of nonpharmacological interventions to reduce sympathetic activity (SA) and cardiac pre-load on MAP and PP.

Methods: Pulse-wave analysis of the radial artery (SphygmoCor AtCor Medical, Sydney, Australia) calibrated from 3 consecutive readings of brachial BP was used for the evaluation of central PP and MAP. Measurements were obtained before and after supervised device-guided paced breathing (DGB), which reduces SA, and lower limb venous occlusion (VO), which decreases cardiac pre-load. Interventions were performed in random order.

Results: In 36 HT patients (16 female) age (mean \pm SD) $46 \pm 12$ years, BP $144.8 \pm 17.7 / 88.2 \pm 10.3 \mathrm{mmHg}$ reduction of brachial BP was greater during DGB compared to VO: (mean (95\% confidence interval)):-9.7(-11.6,-7.8) $\mathrm{mmHg}$ vs $-3.8(-5.8,-1.7) \mathrm{mmHg}$ for systolic BP and $-4.1(-5.6,-2.5) \mathrm{mmHg}$ vs $1.1(-0.1,2.2) \mathrm{mmHg}$ for diastolic BP, respectively, $\mathrm{P}<0.05$. Effects of DGB and VO were similar on PP: $-3.5(-4.8,-2.3) \mathrm{mmHg}$ for DBG and $-3.1(-5.5,-0.7) \mathrm{mmHg}$ for $\mathrm{VO}, \mathrm{P}>0.1$. MAP reduction was only significant during DBG $(-6.4 \quad(-8.1,-4.7)$ $\mathrm{mmHg})$ compared to $\mathrm{VO}(-1.2(-2.7,0.1) \mathrm{mmHg}, \mathrm{P}<0.01$ between the interventions.

Conclusions: Reducing SA via DGB decrease both steady state and pulsatile components of BP while pre-load reduction selectively decreases PP. Treatments that target sympathetic activity and pre-load could potentially be tailored to the weather hypertension is predominantly systolic or diastolic in nature.

Disclosures: None 
P - 38

Is baroreceptor reflex regulation of arterial blood pressure disturbed in young men who are the offspring of hypertensive parents $(\mathrm{OH})$ relative to young men with normotensive parents (ON)?

\section{Emily Jones $^{1}$, Janice Marshall ${ }^{1}$}

\section{${ }^{1}$ University of Birmingham, United Kingdom}

Introduction: $\mathrm{OH}$ have greater risk of hypertension than ON. Increased pressor responsiveness to mental stressors has been reported in young $\mathrm{OH}$, but whether baroreflex sensitivity (BRS) is disturbed is unclear.

Methods: In $7 \mathrm{ON}$ and $6 \mathrm{OH}$ normotensive men (19-23 yrs), we continuously recorded arterial blood pressure (ABP), and R-R interval from the ECG. At rest and during mental stress (MS: Stroop test), we tested the relationship between R$\mathrm{R}$ interval and systolic pressure (SP) during spontaneous increases or decreases in SP (SPI, SPD), and following the $\mathrm{ABP}$ fall induced by squat-stand: these reflect mainly vagal and sympathetic components respectively.

Results: At rest, in ON, BRS was greater during SPI than SPD: $18.2 \pm 0.9$ vs $14.7 \pm 1.5 \mathrm{~ms} / \mathrm{mmHg}(\mathrm{P}<0.05)$. In OH, SPI, SPD were only $12.9 \pm 2.9$ vs $7.6 \pm 1.5 \mathrm{~ms} / \mathrm{mmHg}$ $(\mathrm{P}=0.09) ; \mathrm{OH}$ vs $\mathrm{ON}: \mathrm{P}<0.05$. During squat-stand, BRS was similar in $\mathrm{ON}$ and $\mathrm{OH}(4.3 \pm 0.7$ vs $4.1 \pm 0.8 \mathrm{~ms} /$ $\mathrm{mmHg})$. In ON, MS evoked a decrease in RR interval (0.9 \pm 0.04 vs $0.7 \pm 0.03 \mathrm{~ms}^{*}, *: \mathrm{P}<0.05$ vs control) but no change in ABP $(99 \pm 3.8$ vs $103 \pm 3 \mathrm{mmHg})$, whereas in $\mathrm{OH}$, there was no change in R-R interval $(0.8 \pm 0.07$ vs 0.8 $\pm 0.08 \mathrm{~ms})$, but ABP increased $(103 \pm 8.6$ vs $118 \pm$ $8.9 * \mathrm{mmHg}$ ). Further, with MS, BRS during SPI was decreased in ON $(11.9 \pm 3.3 * \mathrm{~ms} / \mathrm{mmHg})$, not $\mathrm{OH}(17.5 \pm$ $2.8 \mathrm{~ms} / \mathrm{mmHg}$ ), whereas BRS during squat-stand was unchanged in $\mathrm{ON}$ and $\mathrm{OH}(3.8 \pm 0.6 ; 5.0 \pm 1.0 \mathrm{~ms} / \mathrm{mmHg})$.

Conclusions: At rest, the vagal component of the baroreceptor reflex is blunted in young $\mathrm{OH}$ men, but during mental stress, they lack central inhibition of the vagal component of the baroreflex, yet still show greater pressor responsiveness than $\mathrm{ON}$, potentially hastening hypertension.

Disclosures: none
P - 39

Lack of blood pressure-lowering with inorganic nitrate following oral glucose (Lucozade ${ }^{\circledR}$ ) challenge in healthy individuals

\section{Christopher Floyd ${ }^{1}$, Satnam Lidder ${ }^{1}$, Joanne Hunt ${ }^{1}$, Karen McNeill ${ }^{1}$, Andrew Webb ${ }^{7}$ \\ ${ }^{1}$ King's College London, United Kingdom}

Introduction: Patients with/at risk of type 2 diabetes mellitus fail to exhibit a reduction in blood pressure (BP) or pulse wave velocity (PWV) in response to dietary/inorganic nitrate (NO3-) supplementation[1], assumed to be a consequence of chronic hyperglycaemia/hyperinsulinaemia. We hypothesised that acute physiological elevations in glucose/insulin blunt haemodynamic responses to NO3-.

Methods: We conducted an acute, randomised, placebocontrolled, double-blind, cross-over study on the haemodynamic and metabolic effects of potassium nitrate (KNO3) versus potassium chloride (KCl; placebo) administered $1 \mathrm{~h}$ prior to an oral glucose tolerance test $(75 \mathrm{~g}$ glucose administered as Lucozade $^{\oplus}$ (Lucozade Ribena Suntory Ltd, Uxbridge, UK); OGTT) in 33 healthy volunteers; 13 receiving 'high-dose' $(24 \mathrm{mmol} \mathrm{KNO} 3$ or $\mathrm{KCl})$ and 20 receiving 'low-dose' ( $8 \mathrm{mmol})$.

Results: Compared to baseline, the haemodynamic response to an OGTT in the placebo arm was an increase in pulse pressure ( $\mathrm{PP} ; \mathrm{P}=0.007)$ and heart rate $(\mathrm{P}=<0.001)$. There were no significant changes in absolute systolic BP, diastolic BP, PP or PWV for KNO3 versus $\mathrm{KCl}$. High-dose NO3- resulted in an increase in plasma [insulin] versus $\mathrm{KCl}$ $(\mathrm{P}<0.001)$ without a difference in plasma [C-peptide] or [glucose] (both $\mathrm{P}>0.05$ ). Change in $\mathrm{BP}$ did not correlate with metabolic parameters.

Conclusions: In healthy adults, Lucozade $^{\circledR}$ resulted in a lack of BP-lowering effect of NO3- despite elevated plasma [insulin] with high-dose $\mathrm{KNO} 3$ versus placebo. The increase in plasma [insulin] without a corresponding change in [C-peptide] or [glucose] suggests that NO3- reduces insulin clearance.

Disclosures: A.W. is a shareholder of HeartBeet Ltd which manufactures the active and placebo Beet-It ${ }^{\circledR}$ used in this study. Other Authors: 'none'.

\section{References:}

1.Mills, C.E., et al., Reducing Arterial Stiffness Independently of Blood Pressure: The VaSera Trial. J Am Coll Cardiol, 2017. 70(13): p. 1683-1684. 


\section{$P=40$}

\section{Na MRI can detect diuretic-induced changes in skin sodium content}

\section{Kathleen Connolly ${ }^{1}$, Viknesh Selvarajahv ${ }^{1}$, Frank Reimer', Mary McLean ${ }^{1}$, Joshua Kaggie ${ }^{1}$, Carmel M McEneiry ${ }^{1}$, Ferdia Gallagher ${ }^{1}$, Ian B Wilkinson ${ }^{1}$ \\ ${ }^{1}$ University of Cambridge, United Kingdom}

Introduction: Hypertension has been strongly correlated with excessive salt consumption and subsequent accumulation of sodium in the skin. While the mechanisms by which sodium influences blood pressure remain speculative, quantification of skin sodium may be an important biomarker of cardiovascular risk [1]. However, widespread use of this biomarker remains hampered by the invasiveness of current techniques. In this pilot work, we aim to validate $23 \mathrm{Na}$ MRI as a robust, noninvasive method for in vivo skin sodium quantification.

Methods: Seven human volunteers were standardised to a daily salt intake of $<6 \mathrm{~g}$ and treated with either the diuretic Indapamide $(2.5 \mathrm{mg})$ or a placebo for eight days. Skin biopsies and $23 \mathrm{Na}$ MRI scans were taken before and after treatment. Sodium content in the biopsies (Nabiopsy) was quantified using inductively coupled plasma-optical emission spectrometry (ICP-OES) while sodium content in the $23 \mathrm{Na}$ MRI images (NaMRI) was calculated against $\mathrm{NaCl}$-agar standards. The techniques were compared using Pearson's correlation.

Results: Absolute NaMRI values were $80 \%$ lower than Nabiopsy values with no correlation between techniques $(25.9 \pm 6.2$ vs. $121.0 \pm 17.3 \mathrm{mmol} / \mathrm{L}, \mathrm{r}=0.17, \mathrm{p}=0.54)$. However, the percentage change in NaMRI and Nabiopsy pre- vs. post-treatment correlated well $(\mathrm{r}=0.85, \mathrm{p}=0.016)$.

Conclusions: The discrepancy in absolute values suggests $23 \mathrm{Na}$ MRI may only detect a fraction of total sodium. While the poor correlation of singular NaMRI and Nabiopsy values may limit the suitability of $23 \mathrm{Na} \mathrm{MRI}$ for single-point measurements, 23Na MRI may still be useful in assessing changes in an individual's disease progression or response to treatment.

Disclosures: None

References:

[1] Hypertension 2017;70:930-937
P - 41

Measures of vascular health in a cohort of healthy adults stratified by MTHFR genotype

\section{Martina Rooney ${ }^{1}$, Catherine Francis Hughes ${ }^{1}$, Helene McNulty ${ }^{1}$, Michelle Clements ${ }^{1}$, Sean Strain ${ }^{1}$, Mary Ward $^{1}$ \\ ${ }^{1}$ Ulster University, Coleraine, United Kingdom}

Introduction: Hypertension is the leading cause of preventable, premature mortality. A common polymorphism, the C677T mutation in the gene encoding the folate metabolising enzyme methylenetetrahydrofolate reductase (MTHFR), affects $10 \%$ of the UK and Irish populations, and is associated with a $24-87 \%$ increased risk of hypertension across different populations globally. Previous studies at our centre and elsewhere have confirmed significantly higher blood pressure (BP) in hypertensive adults with the variant MTHFR 677TT genotype. The mechanism linking this genetic factor with higher BP is currently unknown, but may involve a nitric oxide (NO)-mediated effect on endothelial function $^{(1)}$. The aim of this study, therefore, was to investigate BP and endothelial function in generally healthy adults stratified by MTHFR genotype.

Methods: Adults aged 18-60 years $(42.3 \pm 10.5$ years), were recruited from workplaces across Northern Ireland and screened for the MTHFR C677T polymorphism. Participants were invited to an appointment where health and lifestyle information, anthropometric measurements, BP (OMRON, Netherlands) and pulse wave velocity (PWV; SphygmoCor, Australia) were assessed.

Results: Preliminary results from a subset $(n=108)$ of the study cohort show that systolic BP is significantly higher in participants with the TT compared to $\mathrm{CC}$ genotype (137.1 $\mathrm{mmHg}$ vs. $128.5 \mathrm{mmHg}, \mathrm{P}=0.033$ ). Similarly, PWV $(7.3 \mathrm{~m} / \mathrm{s}$ vs. $6.4 \mathrm{~m} / \mathrm{s}, \mathrm{P}=0.047)$ and pulse pressure amplification (PPA; $14.45 \mathrm{mmHg}$ vs 7.55 $\mathrm{mmHg}, \mathrm{P}=0.03$ ) were significantly higher in the TT compared to the $\mathrm{CC}$ genotype group.

Conclusions: In this study of apparently healthy adults, individuals with the MTHFR 677TT genotype have elevated BP and poorer endothelial function compared to their age-matched CC genotype counterparts, with potential consequences for CVD risk. Further studies exploring the mechanism linking this genetic factor with hypertension are warranted.

Acknowledgements: We would like to thank the Northern Ireland Clinical Research Facility (NICRF), City Hospital, Belfast.

Disclosures: none.

References: 
1. McNulty H, Strain JJS, Hughes CF et al. Mol Aspects Med. 2017:53:2-9. https://doi.org/10.1016/j.mam.2016.10. 002

\section{P - 42}

Relationships between 24-Hour Pulse Wave Velocity and Blood Pressure Variability in Early Stage CKD

\section{Anna M Price ${ }^{1}$, Lawrence Hopkins ${ }^{1}$, Helen Wheeldon ${ }^{2}$, Manvir K Hayer ${ }^{1,2}$, Charles J Ferro ${ }^{1,2}$, Nicola C Edwards $^{1,2}$, Jonathan $N$ Townend ${ }^{1,2}$}

${ }^{I}$ Queen Elizabeth Hospital Birmingham, United Kingdom,

${ }^{2}$ University of Birmingham, United Kingdom

Introduction: Pulse wave velocity (PWV), a measure of arterial stiffness is a predictor of cardiovascular mortality. Relationships between blood pressure (BP) variability and ambulatory PWV in early chronic kidney disease (CKD) are unclear.

Methods: Ambulatory blood pressure (ABPM) recordings with Mobil-O-Graph NG; IEM were reviewed in patients with early stage CKD with controlled hypertension and healthy controls.

Results: The 41 patients with early CKD had a lower eGFR 55 vs $83, \mathrm{p}=0.003$, and higher office systolic BP 129 vs $122 \mathrm{p}=0.008$ than 31 healthy controls. Ambulatory $\mathrm{PWV}$, systolic and diastolic BP was significantly higher in those with CKD. Average real variability in systolic BP was higher in early CKD (11.52 vs 9.92, $\mathrm{p}=0.039)$. See Table 1.

Increasing age and CKD status were able to account for $82 \%$ of the variance in ambulatory PWV $(\mathrm{p}<0.05)$. Using forward regression and including both $24 \mathrm{~h}$ systolic and diastolic blood pressure the predictive value of CKD status on ambulatory PWV is lost.

Conclusions: Even with controlled hypertension, patients with early CKD have a greater BP variability and ambulatory PWV. Average BP however, remains the greatest predictor of ambulatory PWV.

Disclosures: None

Table 1 [P - 42]: ABPM recordings.

\begin{tabular}{lcll}
\hline CKD & $n=$ Controls & $n$ & $\mathrm{P}$ \\
41 & $=36$ & value \\
\hline
\end{tabular}

$24 \mathrm{~h}$

$\begin{array}{llll}\text { Systolic BP }(\mathrm{mmHg}) & 126.60 \pm & 119.36 \pm & \mathbf{0 . 0 0 9} \\ & 13.18 & 10.17 & \\ & & 73.94 \pm 8.13 & \mathbf{0 . 0 0 7}\end{array}$

Table (continued)

\begin{tabular}{|c|c|c|c|}
\hline & $\begin{array}{l}\text { CKD } n= \\
41\end{array}$ & $\begin{array}{l}\text { Controls } \\
=36\end{array}$ & $\begin{array}{l}\mathrm{P} \\
\text { value }\end{array}$ \\
\hline & $\begin{array}{l}79.48 \pm \\
9.19\end{array}$ & & \\
\hline $\begin{array}{l}\text { Central systolic BP } \\
(\mathrm{mmHg})\end{array}$ & $\begin{array}{l}115.09 \pm \\
12.55\end{array}$ & $\begin{array}{l}110.27 \pm \\
9.63\end{array}$ & 0.065 \\
\hline $\begin{array}{l}\text { Central diastolic BP } \\
(\mathrm{mmHg})\end{array}$ & $\begin{array}{l}81.02 \pm \\
9.90\end{array}$ & $75.94 \pm 7.94$ & 0.016 \\
\hline $\mathrm{PWV}(\mathrm{m} / \mathrm{s})$ & $7.67 \pm 1.76$ & $6.90 \pm 1.30$ & 0.034 \\
\hline
\end{tabular}

P - 43

Forearm vasoconstriction during mental stress is associated with non-dipping circadian blood pressure pattern in young adult White Europeans (WEs) and Black Africans (BAs): an early indicator of
cardiovascular disease?

\section{$\underline{\text { Abimbola Aiku }^{1}, \text { Una Martin }}{ }^{1}$, Janice Marshall ${ }^{1}$ \\ ${ }^{1}$ University of Birmingham, United Kingdom, ${ }^{2}$ University of Ibadan, Nigeria}

Introduction: BAs have greater risk of developing hypertension than WEs. Among middle-aged WE hypertensives, nocturnal non-dipping is associated with endothelial dysfunction. Higher prevalence of endothelial dysfunction and nocturnal non-dipping have been documented in BAs than WEs. In young adults, exaggerated vasoconstrictor responses to novel stimuli are predictive of future hypertension.

We hypothesized that non-dipping WEs and BAs show blunted reactive hyperaemia (endothelium-dependent) and smaller forearm vasodilatation in response to repetition of environmental stressors (5 sounds: S1-5; $100 \mathrm{~dB}, 2 \mathrm{KHz}$ ) than dippers.

Methods: In 17 WEs and 16 BAs (18-26 years), arterial blood pressure (ABP) was continuously recorded by photoplethysmography, forearm blood flow (FBF) by venous occlusion plethysmography; forearm vascular conductance (FVC): FBF/ABP. $24 \mathrm{~h}$ ambulatory blood pressure monitoring was done at $30 \mathrm{~min}$ intervals from $7 \mathrm{am}-11 \mathrm{pm}$ and hourly from $11 \mathrm{pm}-7 \mathrm{am}$.

Results: BAs had higher $24 \mathrm{~h}$ SBP than WEs $(117 \pm 3.17$ vs $110 \pm 1.33 \mathrm{mmHg} ; \mathrm{p}<0.05)$ and higher proportion of pre-/hypertensives $(7 / 16,40 \%$ vs $1 / 15,6 \%) .40 \%$ of BAs were non-dippers vs $17.6 \%$ of WEs. Peak reactive hyperaemia was not different between dippers and non-dippers 
(WEs: $+0.39 \pm 0.03$ vs $+0.45 \pm 0.08 ;$ BAs: $+0.41 \pm 0.05$ vs $+0.34 \pm 0.05 \mathrm{ml}$.min. $100 \mathrm{~g}-1: \mathrm{p}>0.05$ in each ethnicity). However, over S1-S5, dippers showed a net increase in FVC (WEs: $+0.005 \pm 0.002$; BAs: $+0.007 \pm 0.002$ conductance units (CU), non dippers, a net decrease in FVC (WEs: $-0.007 \pm 0.004$; BAs: $-0.007 \pm 0.003 \mathrm{CU}$ ): $\mathrm{P}<0.05$ dippers vs non-dippers in each ethnicity).

Conclusions: We propose that in young normotensive WEs and BAs, a non-dipping night-time $\mathrm{ABP}$ profile together with forearm vasoconstriction to mental stress may precede endothelial dysfunction and provide an early indication of future hypertension.

Disclosures: none

\section{P - 44}

\section{High flavanol-containing chocolate improves endothelium-dependent vasodilator responses in healthy, normotensive men}

\section{$\underline{\text { Hassan Latif }}{ }^{1}$, Abimbola Aiku ${ }^{1}$, Janice Marshall ${ }^{1}$ \\ ${ }^{1}$ University of Birmingham, United Kingdom}

Introduction: Dietary flavanols have beneficial cardiovascular effects, including reducing arterial blood pressure (ABP) in hypertensives and improving flow-mediated dilatation. Increased nitric oxide (NO) availability and decreased cyclooxygenase inhibition of NO synthase have been implicated. Whether flavanols affect tissue vascular responses is unclear.

Methods: In 10 normotensive men (19-26 years), we recorded ABP continuously by photoplethysmography, forearm blood flow (FBF) with venous occlusion plethysmography and cutaneous perfusion with laser Doppler flowmetry. We tested responses evoked by arterial occlusion for 3 min (reactive hyperaemia: $\mathrm{RH}$ ), iontophoresis of acetylcholine (ACh) by 7 pulses at $0.1 \mathrm{~mA}, 8$ th at $0.2 \mathrm{~mA}$ at $1 \mathrm{~s}$ intervals and by mental stress (MS; Stroop test) for 3 min, before and following dark chocolate (DC) for 3 days (396 mg flavanols/day p.o.)

Results: DC had no effect on mean ABP by sphygmomanometer $(88 \pm 2$ vs $87 \pm 2 \mathrm{mmHg}$ ), or $\mathrm{RH}$ in forearm (peak $41.6 \pm 3.2 \mathrm{vs} 40.2 \pm 2.7 \mathrm{ml} / 100 \mathrm{~m} / \mathrm{min}$ ), but tended to augment $\mathrm{RH}$ in cutaneous circulation $(106.2 \pm 10.2$ vs $139.8 \pm 1.7 \mathrm{PU} ; \quad \mathrm{P}=0.07)$ and augmented ACh-evoked dilatation (mean response: $86.2 \pm 11.7 \mathrm{PU}$ vs $145.7 \pm$ 12.0PU, $\mathrm{P}<0.001)$. Further, $\mathrm{DC}$ attenuated the mean increase in $\mathrm{ABP}$ evoked by MS (to $84 \pm 1$ vs $80 \pm 2 \mathrm{mmHg}$; $\mathrm{P}<0.001)$ and augmented the increase in forearm vascular conductance (FBF/ABP: $0.06 \pm 0.004$ vs $0.076 \pm 0.003 \mathrm{CU}$; $\mathrm{P}<0.01)$.
Conclusions: Even in healthy young men, dietary flavanols can augment RH and ACh-evoked endotheliumdependent vasodilator responses in skin, whilst augmenting forearm vasodilatation and limiting the increase in $\mathrm{ABP}$ evoked by mental stress. Since NO makes little active contribution to $\mathrm{RH}$ in muscle, these findings may be explained by flavanol-induced increase in NO availability.

Disclosures: None

\section{P - 45 \\ Breaking up prolonged sitting with simple resistance activities improves arterial function in overweight/obese adults}

\section{Rachel Climie ${ }^{1}$, Michael Wheeler ${ }^{2}$, Megan Grace ${ }^{2}$, Elizabeth Lamber', Neale Cohen ${ }^{2}$, Neville Owen ${ }^{2}$, Bronwyn Kingwell2, David Dunstan ${ }^{2}$, Danny Green ${ }^{3}$ \\ ${ }^{1}$ Inserm, Paris, France, ${ }^{2}$ Baker Heart and Diabetes Institute, Melbourne, Australia, ${ }^{3}$ University of Western Australia, Perth, Australia}

Introduction: Prolonged sitting contributes to cardiovascular disease (CVD) risk. The underlying mechanisms are unknown, but may include changes in arterial function and vasoactive proteins. We examined this, compared to interruptions to sitting, in adults at increased CVD risk.

Methods: In a randomized crossover trial, 19 sedentary overweight/obese adults (mean \pm SD $57 \pm 12$ yrs), completed two laboratory-based conditions: five hours uninterrupted sitting (SIT) and; five hours sitting interrupted every 30 min by three minutes of simple resistance activities (SRA). Femoral artery function (flow mediated dilation; FMD), blood flow and shear rate were measured at zero hour, 30 min, one, two and five hours. Brachial FMD was assessed at zero and five hours. Plasma was collected hourly for measurement of endothelin-1 (ET-1), nitrates/nitrites, vascular cell adhesion molecule-1 (VCAM-1) and intercellular adhesion molecule-1 (VCAM-1). Data are presented as marginal means and $95 \%$ confidence intervals.

Results: There was a significant decline in femoral artery FMD, averaged over five hours in the SIT condition, relative to SRA ( $\mathrm{p}<0.001)$. Plasma ET-1 total AUC over five hours increased in the SIT condition compared to SRA ( $p=$ 0.006). There was no difference between conditions in femoral shear rate, blood flow, brachial FMD, nitrates/ nitrites, VCAM-1 or ICAM-1 ( $p>0.05$ for all).

Conclusions: Relative to regular interruptions to sitting time, five hours of prolonged sitting impaired femoral artery vasodilator function and increased ET-1 in overweight/ obese adults. There is the need to build on this evidence 
from an acute study, to better understand the longer-term adverse vascular-related consequences of prolonged sitting.

Disclosures: None

\section{$P$ - 46 \\ An assessment of aortic stiffness indices using a model of healthy cardiovascular ageing}

\section{$\underline{\text { Peter Charlton }^{1}, \text { Phil Chowienczyk }}{ }^{1}$, Jordi Alastruey ${ }^{1}$ \\ ${ }^{1}$ King's College London, King's Health Partners, London, United Kingdom}

Introduction: Over 30 arterial stiffness indices (ASIs) have been designed to assess arterial stiffness from pulse waves. However, their performances have not been directly compared, and there is evidence that they are influenced by other cardiovascular properties. Our aim was to assess their performance using a model of healthy cardiovascular ageing.

Methods: The haemodynamics of a young subject were modelled using a numerical model of blood flow. Model parameters were varied to create a population of virtual subjects aged 25 to 75 exhibiting normal physiological variation. Blood pressure and pulse oximetry pulse waves were simulated for each subject. The model was verified through comparison with in vivo measurements. ASIs were calculated using pulse wave analysis techniques. Their performances were assessed through comparison with reference aortic stiffness values using correlation and limits of agreement techniques.

Results: Most ASIs were indicative of aortic stiffness, although many were also influenced by other arterial parameters, including arterial diameter and the stiffness of other arteries, and by cardiac parameters including heart rate and stroke volume. ASIs derived from the second derivative of the pulse wave were less influenced by heart rate, but more strongly influenced by the stiffness of smaller arteries.

Conclusions: The performances of over 30 ASIs were assessed at multiple arterial sites. Performance could be improved by combining ASIs to reduce the influence of other cardiovascular properties. In the future, the database of pulse waves could be used to assess techniques for non-invasive estimation of central blood pressure and cardiac output.

Disclosures: none

\section{$P$ - 47}

\section{Screening for hypertension using retinal vascular calibre in ultra-widefield fundus imaging}

\author{
Gavin Robertson ${ }^{1}$, Alan Fleming ${ }^{1}$, Michelle $C$ \\ Williams' ${ }^{2}$, Emanuele Trucco ${ }^{3}$, Nicola Quinn ${ }^{4}$, Ruth E
}

$\mathrm{Hogg}^{4}$, Gareth J Makay ${ }^{4}$, Frank Kee ${ }^{4}$, lan S Young ${ }^{4}$, Enrico Pellegrini ${ }^{1}$, David E Newby ${ }^{2}$, Edwin J R van Beek $^{2,5}$, Tunde Peto ${ }^{4}$, Baljean Dhillon ${ }^{6}$, Jano van Hemert $^{1}$, Tom MacGillivray,

${ }^{1}$ Optos PLC, Dunfermline, United Kingdom, ${ }^{2}$ Center of Cardiovascular Science, University of Edinburgh, United Kingdom, ${ }^{3}$ The VAMPIRE project, Computer Vision and Image Processing Group, School of Science and Engineering, University of Dundee, United Kingdom, ${ }^{4}$ Centre for Public Health, Queen's University Belfast, United Kingdom, ${ }^{5}$ Edinburgh Imaging Facility QMRI, University of Edinburgh, United Kingdom, ${ }^{6}$ The VAMPIRE project, Centre for Clinical Brain Sciences, University of Edinburgh, United Kingdom

Introduction: Changes to the retinal vasculature are associated with systemic hypertension independent of traditional risk factors. We investigate whether measurements of retinal vascular calibre from ultra-widefield fundus imaging are associated with hypertensive status.

Methods: We automatically identified retinal vessels and classified them as arteriole or venule using machine learning and image processing techniques in a sub-set of subjects, aged 50-59 years, enrolled in the Northern Ireland Cohort of Longitudinal Ageing (School of Medicine, Dentistry and Biomedical Sciences Ethics Committee, Queen's University Belfast, SREC 12/23). Vascular calibre in the peripheral retina was measured to calculate the arteriole-venule ratio (AVR). Subjects were categorised as normotensive or hypertensive, according to thresholds on systolic/diastolic blood pressure measurement $(140 / 90 \mathrm{~mm} \mathrm{Hg})$ averaged over two sitting blood pressure measurements in a clinical setting.

Results: Left eyes were analysed from 431 subjects (mean age of $54.5 \pm 2.8$ years; 239 (55.5\%) females), with $148(34.3 \%)$ subjects categorised as hypertensive. Using a two-sample t-test, arterioles were thinner $(\mathrm{p}<0.005,95 \%$ confidence interval $[\mathrm{CI}],-9 \mu \mathrm{m},-4 \mu \mathrm{m})$ and AVR was smaller ( $\mathrm{p}<0.005,95 \% \mathrm{CI},-0.08,-0.04)$ in subjects with hypertension. The area under a receiver operator characteristic curve of AVR for hypertensive status was 0.67 (95\% CI, 0.61 to 0.72 ).

Conclusions: In this study, automated measurement of AVR in ultra-widefield fundus imaging was associated with hypertension. With further development, such as evaluation against diagnosis of hypertension obtained from ambulatory blood pressure monitoring clinics, this system could become a test for undiagnosed hypertension in people attending routine eye health check-ups.

Disclosures: GR, AF, EP and JvH are employees of Optos PLC, NQ, REH, TP, TM, BD and MT have received funding from Optos PLC 


\section{P - 48 Omitted}

P - 49

Endothelial microparticles from VEGF inhibitors (VEGFi)-treated cancer patients mediate endothelial cells signaling and ET-1 production: implications in VEGFi-induced hypertension

\section{Karla Neves ${ }^{1}$, Francisco Rios ${ }^{1}$, Rheure Lopes ${ }^{1}$, Martin McLeod ${ }^{2}$, Judith Dixon Hughes ${ }^{3}$, Robert Jones ${ }^{4}$, Augusto Montezano ${ }^{1}$, Jeff Evans ${ }^{4}$, Rhian Touyz ${ }^{1}$ \\ ${ }^{1}$ Institute of Cardiovascular and Medical Sciences, University of Glasgow, United Kingdom, ${ }^{2}$ The \\ Experimental Cancer Medicine Centre (ECMC), Glasgow, United Kingdom, ${ }^{3}$ Cancer Research UK Glasgow Clinical Trials Unit, United Kingdom, ${ }^{4}$ Institute of Cancer Sciences, Cancer Research UK Beatson Institute, University of Glasgow, United Kingdom}

Introduction: Vascular endothelial growth factor receptor inhibitors (VEGFRi), used as anti-angiogenic drugs to treat cancer, cause hypertension where mechanisms are still not fully elucidated. Microparticles (MPs) are biomarkers of cell damage. MPs also act as biovectors and influence vascular cell signalling and function. We questioned whether plasma levels of endothelial MPs from cancer patients treated with VEGFi are elevated (possibly reflecting underlying vascular injury) and whether these MPs influence endothelial cell signalling.

Methods: Plasma MPs were isolated from cancer patients before and after treatment with VEGFRi (sunitinib, pazopanib or sorafenib). Human aortic endothelial cells (HAEC) were stimulated with isolated MPs (106 MPs/mL). MPs were characterized by flow cytometry; protein and gene expression by immunoblotting and qPCR; ROS and NO production by lucigenin and immunofluorescence.

Results: VEGFRi treatment increased plasma endothelial cells-derived MPs ( 1.5 fold; $p<0.05$ vs before treatment). HAEC exposed to post-treatment MPs increased prepro-ET-1 mRNA (9.1 fold; $\mathrm{p}<0.01$ ). Post-treatment MPs also increased ROS generation (5 min: 1.2 fold, $\mathrm{p}<0.01$; 30 min: 1.6 fold, $\mathrm{p}<0.01$; 60 min: 1.8 fold, $\mathrm{p}<0.05$ ) and phosphorylation of the eNOS inhibitory site (Thr495) in HAEC (1.8 fold; $\mathrm{p}<0.05)$. NO levels in HAEC exposed to MPs were decreased (1.2 fold; $p<0.05)$, an effect blocked by an ETB antagonist. Inflammatory marker gene expression was increased only in HAEC exposed to post-treatment MPs $(\mathrm{p}<0.01)$.

Conclusions: VEGFi treatment causes an increase in circulating MPs and that play a role in endothelial cell signalling and ET-1 production. These molecular processes may contribute to vascular dysfunction and hypertension in VEGFi-treated cancer patients.

Disclosures: None.

\section{P - $\mathbf{5 0}$ \\ Vascular Endothelial Growth Factor Inhibitor (VEGFI) Chemotherapy Disturbs Endothelial Cell Signalling and Survival Pathways}

\author{
Alan Cameron ${ }^{1}$, Karla Neves ${ }^{1}$, Francisco Rios ${ }^{1}$, Augusto \\ Montezano ${ }^{1}$, Ninian Lang ${ }^{1}$, Rhian Touyz ${ }^{1}$ \\ ${ }^{1}$ Institute of Cardiovascular and Medical Sciences, \\ University of Glasgow, United Kingdom
}

Introduction: Hypertension affects 40-60\% of patients treated with vascular endothelial growth factor inhibitor (VEGFI) chemotherapy. The underlying molecular mechanisms are elusive but vascular dysfunction may be important. We questioned whether VEGFI influences proinflammatory and stress signalling pathways in human endothelial cells.

Methods: Human aortic endothelial cells (HAECs) were exposed to VEGF and the VEGFI Vatalanib for $5 \mathrm{~min}, 15$ min and $24 \mathrm{~h}$. Effects on endothelial nitric oxide synthase (eNOS) $\operatorname{Ser}^{1177}$ (activation site) phosphorylation, stressrelated kinases (ERK 1/2 and JNK) and the pro-survival kinase, Akt, were assessed by Western blot analysis. Realtime PCR was performed to probe for mediators of vasoconstriction (pre-pro-ET-1), inflammation (VCAM-1, ICAM-1, IL-6, TNF- $\alpha$, MCP-1) and thrombosis (vWF, tPA, PAI-1, TF, PGI2, PAFR). Data were analysed by Student's t-test.

Results: VEGF and Vatalanib reduced phosphorylation of ERK 1/2 $(36 \%, \mathrm{p}<0.01)$ and Akt $(33 \%, \mathrm{p}=0.03)$. After $24 \mathrm{~h}$, the mRNA expression of IL-6 $(97 \%, \mathrm{P}<0.01)$ and MCP-1 $(91 \%, \mathrm{p}<0.01)$ was reduced, while levels of VCAM-1, ICAM-1, TNF- $\alpha$ and the thrombotic marker mRNA expression were unchanged. There was a trend towards reduced eNOS phosphorylation after $15 \mathrm{~min}(42 \%$, $\mathrm{p}=0.06)$ and increased pre-pro-ET1 mRNA expression after $24 \mathrm{~h}(21 \%, \mathrm{p}=0.08)$.

Conclusions: VGEFI chemotherapy reduces stress and pro-survival kinase phosphorylation, and may contribute to reduced eNOS phosphorylation and increased pre-pro-ET-1 expression. Our findings indicate that VEGFI influences endothelial signalling promoting vascular injury and dysfunction, processes which may contribute to VEGFIinduced hypertension.

Disclosures: None 


\section{$P$ - 51}

Role of TRPM2 in redox signalling and vascular biology in hypertension

\section{Rheure Alves-Lopes ${ }^{1}$, Karla Neves ${ }^{1}$, Aikaterini Anagnostopoulou ${ }^{1}$, Silvia Lacchini ${ }^{1}$, Augusto Montezano ${ }^{1}$, Rhian Touyz ${ }^{1}$}

${ }^{1}$ University of Glasgow, United Kingdom

Introduction: $\mathrm{ROS}$ regulates $\mathrm{Ca} 2+$ homeostasis by mechanisms still not fully understood. TRPM2, a redoxsensitive channel permeable to $\mathrm{Ca} 2+$ and $\mathrm{Na}+$, is activated by $\mathrm{H} 2 \mathrm{O} 2$ via oxidation or ADPR production. We tested the hypothesis that TRPM2 is a molecular target of ROS leading to regulation of $\mathrm{Ca} 2+$ levels and vascular function in hypertension.

Methods: VSMCs from normotensive and hypertensive subjects were used. $\mathrm{Ca} 2+$ and $\mathrm{Na}+$ influx was assessed by fluorescence microscopy.

Results: O2- (1.65 fold) and $\mathrm{H} 2 \mathrm{O} 2$ (1.88 fold) generation were enhanced in VSMCs from hypertensive subjects (p < 0.05). $\mathrm{Ca} 2+$ (AUC: 1.36 fold) and $\mathrm{Na}+$ influx (AUC: 1.2 fold) were also increased in cells from hypertensive subjects $(\mathrm{p}<0.05)$, an effect blocked by PEG-catalase and, 2-APB, 8-br or olaparib (TRPM2 inhibitors). Additionally, in the absence of $\mathrm{Na}+,[\mathrm{Ca} 2+] \mathrm{i}$ was reduced to levels detected in cells from normotensive subjects. TRPM2induced $\mathrm{Na}+$ influx may influence the sodium-calcium exchanger (NCX), which in reverse mode, promotes $\mathrm{Ca} 2+$ influx. Benzamil, KB-R7943 and YM244769 (NCX inhibitors) also ameliorated the increased $\mathrm{Ca} 2+$ influx observed in hypertension. MLC20 activation, a marker of cell contraction, was increased in VSMCs from hypertensive subjects (1.72 fold), an effect reversed by TRPM2 and NCX inhibitors $(p<0.05)$. Arteries from hypertensive mice exhibit increased TRPM2 (1.5 fold) and NCX (1.9 fold) mRNA expression and increased U46619-induced vasoconstriction (Emax: $7.6 \pm 0.2$ vs $10.21 \pm 0.3$ ), effect reversed by TRPM2 and NCX inhibitors $(\mathrm{p}<0.05)$.

Conclusions: In conclusion, activation of redoxdependent TRPM2 channel contributes to increased calcium and sodium influx, partially through NCX activation. The results highlight a novel pathway linking ROS to $\mathrm{Ca} 2$ + and $\mathrm{Na}+$ signalling through TRPM2/NCX, leading to vascular dysfunction in hypertension.

Disclosures: None
$P-52$

Nox 5 regulation of vascular contraction involves oxidation of endoplasmic reticulum calcium channels and calreticulin

\section{Augusto Montezano ${ }^{1}$, Livia De Lucca Camargo ${ }^{1}$, Emma Carrick', Francisco Rios', Rhian Touyz ${ }^{1}$ \\ ${ }^{1}$ University of Glasgow, United Kingdom}

Introduction: The biological function of calcium-sensitive Nox5 is unknown. The aim of the study is to define the role of Nox 5 in contraction.

Methods: Human arteries and mice expressing human Nox 5 in smooth muscle (Nox $5+$ SM22+) were studied and used for VSMC culture.

Results: In arteries from hypertensive subjects, Nox5 expression was increased (50\%, $\mathrm{p}<0.05$ vs control). In human VSMCs, AngII-induced ROS generation (1 fold) and activation of myosin light chain (MLC) (2.5 fold) was exacerbated in cells from hypertensive subjects ( $p<0.05$ vs control); an effect blocked by Nox 5 siRNA. Contraction to $\mathrm{U} 46619$ was increased in Nox $5+/ \mathrm{SM} 22+(5.8 \pm 0.3 \mathrm{mN}$ vs WT: $4.2 \pm 0.2 \mathrm{mN}, \mathrm{p}<0.05)$, an effect blocked by a NAC (ROS scavenger), calmidazolium (calmodulin inhibitor), dantrolene (ryanodine receptor $\mathrm{Ca} 2+$ channel inhibitors) and CDN1163 (SERCA channel activator), but not by a Nox1/Nox4 inhibitor (GKT137831). ONOO- levels were increased in vessels from Nox $5+/ \mathrm{SM} 22+(5.8 \pm 0.9$ vs WT $3.4 \pm 0.1 \mathrm{AU} / \mathrm{mg}, \mathrm{p}<0.05)$. Inactivation of MYPT1 $(181 \pm 1.8 \mathrm{AU}$ vs $164 \pm 1.9 \mathrm{AU}$ WT $)$ and activation of MLC $(207 \pm 10.3 \mathrm{AU}$ vs $155 \pm 2.7 \mathrm{AU}$ WT $)$ were increased in VSMCs from Nox $5+$ SM $22+(\mathrm{p}<0.05)$. In VSMCs from Nox $5+$ SM $22+$ where reversibly oxidized proteins were immunoprecipitated, we observed oxidation of Nox5, decreased oxidation of MYPT1 and increased oxidation of SERCA2b. Proteome analysis of human VSMCs identified the ER $\mathrm{Ca} 2$ + sensor, calreticulin, as a potential Nox 5 biding protein. Calreticulin reversible oxidation was increased in VSMCs from Nox $5+\mathrm{SM} 22+$ mice and hypertensive subjects.

Conclusions: Our one crosstalk between redox signalling and $\mathrm{Ca} 2+$ in the vasculature, where Nox 5 regulation of contraction is dependent of ROS, $\mathrm{Ca} 2+$ and endoplasmic reticulum localized $\mathrm{Ca} 2+$ channels/proteins.

Disclosures: None 


\section{P - 53}

Interplay between Nox-regulated oxidative stress and ER stress response in experimental hypertension

\section{Livia De Lucca Camargo ${ }^{1}$, Adam Harvey ${ }^{1}$, Francisco Rios $^{1}$, Delyth Graham ${ }^{1}$, Richard Hartley ${ }^{1}$, Neil Bulleid ${ }^{1}$, Augusto Montezano ${ }^{1}$, Rhian Touyz ${ }^{1}$}

${ }^{1}$ University of Glasgow, United Kingdom

Introduction: Nox-derived ROS and ER stress are implicated in vascular dysfunction in hypertension. However, the mechanisms involved in these processes are still unknown.

Methods: We investigated how oxidative stress impacts the oxidative proteome and the ER stress response in VSMCs from SHR rats.

Results: High levels of ROS were observed in SHR, measured by chemiluminescence $(67.1 \%)$ and amplex red (204\%), p $<0.05$ vs WKY. Reversible protein oxidation (sulfenylation) and irreversible protein tyrosine phosphatase (PTP) and peroxiredoxin (Prx) oxidation were also increased in SHR $(\mathrm{p}<0.05$ vs WKY). ER stress response was upregulated in SHR, as phosphorylation of ER stress sensors PERK and IRE1 $\alpha$, as well as expression of chaperones $\mathrm{BiP}$ were increased ( $\mathrm{p}<0.05$ vs WKY). Cell fractionation revealed that Noxs were upregulated in a compartment-specific manner in SHR: Nox1 in plasmamembrane (1.6 fold) and Nox4 in ER (1.3 fold), p $<0.05$ vs WKY. Inhibition of Nox1 (NoxA1ds) and Nox1/4 (GKT137831) normalized vascular ROS generation and protein oxidation in SHR. Using a biotin-tagged dimedonebased probe (DCP-Bio) we observed increased oxidation of IRE1 $\alpha \quad(63 \%)$ in SHR, $\mathrm{p}<0.05$. Treatment with GKT137831 reduced IRE1 $\alpha$ sulfenylation, while NoxA1ds reduced PERK phosphorylation, $\mathrm{p}<0.05$. Inhibition of ER stress (4-PBA) reduced ROS levels and expression of Nox4 in SHR. Increased VSMC proliferation in SHR measured by FACS $(51 \%, \mathrm{p}<0.05$ vs WKY) was normalized by GKT137831 and 4-PBA. Vascular hypercontractility, assessed in mesenteric arteries by wire myography, was attenuated by 4-PBA.

Conclusions: Our findings demonstrate that in hypertension oxidative stress is associated with ER stress through processes involving Nox decompartmentalization, where Nox1-mediated activation of PERK and Nox4-induced oxidation of IRE1 $\alpha$.
Disclosures: None

\section{P - 54 \\ Cardiovascular inflammation and fibrosis in TRPM7 kinase deficient mice}

\section{Francisco Rios ${ }^{1}$, Zou Zhi-Guo ${ }^{1}$, Livia De Lucca Camargo $^{1}$, Augusto Montezano ${ }^{1}$, Rhian Touyz ${ }^{1}$ \\ ${ }^{1}$ University of Glasgow, United Kingdom}

Introduction: TRPM7 is a channel fused to $\alpha$-kinase domain permeable to $\mathrm{Mg} 2+$. TRPM7 deficiency leads to increased blood pressure and inflammation. Here we tested whether TRPM7 is protective against cardiac injury by modulating the inflammatory responses.

Methods: Wild-type (WT) and heterozygote TRPM7 (TRPM7+/-) mice were used. Inflammatory cell migration was studied by intravital microscopy. Cardiac function was assessed by echocardiogram. Immune cell populations were characterized by flow cytometry. Bone-marrow derived macrophages (BMDM) and Cardiac fibroblasts (CF) were obtained from WT and TRPM7+/-.

Results: $+/-$. Intravital microscopy revealed that in TRPM7+/- mice, leukocytes exhibit reduced velocity $(47 \%)$, increased adhesion (222\%) and increased transmigration $(480 \%)$, versus WT $(\mathrm{p}<0.05)$. Vessels from TRPM7+/- showed increased VCAM-1 expression (33fold) vs WT. TRPM7+/- presented increased plasma levels of the cardiac fibrosis marker galectin-3 (Gal-3) (2.5 $\pm 0.3 \mathrm{ng} / \mathrm{mL}$ vs WT: $1.5 \pm 0.2 \mathrm{ng} / \mathrm{mL}), \mathrm{p}<0.05$. Hearts from TRPM7+/- mice showed increased Gal-3 $(16.6 \pm 3.6$ vs WT $9.2 \pm 1.2$ cells/field), fibrotic area $(6.7 \%$ vs WT $2.6 \%)$, fibronectin (2.8-fold), and reduced E/A ratio 30\%, $\mathrm{p}<0.05$. Cardiac macrophages from TRPM7+/- presented $45 \%$ reduction of intracellular $\mathrm{Mg} 2+$ and high CD206 expression (30\% vs WT 14\%), $\mathrm{p}<0.05$. BMDM macrophages from TRPM7 $+/-$ mice presented increased Gal-3 (2.6 \pm $0.05 \mathrm{ng} / \mathrm{mL}$ vs WT $2.1 \pm 0.09 \mathrm{ng} / \mathrm{mL}), \mathrm{p}<0.05$. Treatment of CF with macrophage supernatant from TRPM7+lexhibited increased fibronectin (43\%) and PCNA (36\%) (p $<0.05)$, effects that were normalised by $\mathrm{Mg} 2+$ treatment.

Conclusions: In conclusion, TRPM7 deficiency is associated with cardiovascular inflammation and fibrosis, through processes that are $\mathrm{Mg} 2+-$ dependent.

Disclosures: None 